\title{
PRO-GROUPS AND GENERALIZATIONS OF A THEOREM OF BING
}

\author{
ALEX CLARK, STEVEN HURDER, AND OLGA LUKINA
}

\begin{abstract}
A matchbox manifold is a generalized lamination, which is a continuum whose path components define the leaves of a foliation of the space. A matchbox manifold is $M$-like if it has the shape of a fixed topological space $M$. When $M$ is a closed manifold, in a previous work, the authors have shown that if $\mathfrak{M}$ is a matchbox manifold which is $M$-like, then it is homeomorphic to a weak solenoid. In this work, we associate to a weak solenoid a pro-group, whose pro-isomorphism class is an invariant of the homeomorphism class of $\mathfrak{M}$. We then show that an $M$-like matchbox manifold is homeomorphic to a weak solenoid whose base manifold has fundamental group which is non co-Hopfian; that is, it admits a non-trivial self-embedding of finite index. We include a collection of examples illustrating this conclusion.
\end{abstract}

\section{InTRODUCTION}

A continuum is a compact connected metric space. From the very beginnings of the subject of Topology in the first half of the twentieth century, examples of continua with unusual properties were a source of motivation for the development of the subject. For example, the Warsaw circle, the Sierpinsky carpet [3], and the 1-dimensional solenoids introduced by Vietoris [79] and van Danzig [75] are all well-known examples of "non-trivial" continua. Continua frequently arise as invariant sets for dynamical systems, and moreover as quoted from Barge and Kennedy [7:

One can argue quite convincingly that continuum theory first arose from problems in dynamics even before there was a definition of a topological space.

The study of the invariant continua for a dynamical system is a basic technique for the analysis of its global dynamics. There are many established approaches for the study of continua, as discussed in the book by Sam Nadler [62]. One of these is the following fundamental result of Hans Freudenthal:

THEOREM 1.1 (Freudenthal [41]). Let $\mathfrak{M}$ be a continuum, then there exists a sequence of maps

$$
\mathcal{P}=\left\{f_{\ell+1}: X_{\ell+1} \rightarrow X_{\ell} \mid \ell \geq 0\right\}
$$

where for each $\ell \geq 0, X_{\ell}$ is a finite connected polyhedron and each map $f_{\ell+1}$ is a continuous surjection, such that $\mathfrak{M}$ is homeomorphic to an inverse limit,

$$
\Phi_{\mathcal{P}}: \mathfrak{M} \cong \mathcal{S}_{\mathcal{P}} \equiv \lim _{\longleftarrow}\left\{f_{\ell+1}: X_{\ell+1} \rightarrow X_{\ell} \mid \ell \geq 0\right\}
$$

The maps $f_{\ell+1}$ in (1) are called the bonding maps of the presentation $\mathcal{P}$.

The inverse limit is considered as a subset of the Cartesian product of the spaces $\left\{X_{\ell} \mid \ell \geq 0\right\}$ with the Tychonoff topology, and a point is represented by a sequence $x=\left(x_{\ell}\right)_{\ell \geq 0}$ where $x_{\ell} \in X_{\ell}$ and $f_{\ell+1}\left(x_{\ell+1}\right)=x_{\ell}$ for $\ell \geq 0$. A presentation $\mathcal{P}$ is said to be trivial if all of the maps $f_{\ell+1}$ are homeomorphisms, and thus $\mathfrak{M}$ is homeomorphic to a finite connected polyhedron.

Observe that given a presentation $\mathcal{P}$ for a continuum $\mathfrak{M}$ as in (2), for all $\ell \geq 0$, there is a continuous surjection $\Pi_{\ell}: \mathfrak{M} \rightarrow X_{\ell}$ given by $\Pi_{\ell}\left(\left(x_{\ell}\right)_{\ell \geq 0}\right)=x_{\ell}$. It then follows that for any $\epsilon>0$, there exists some $\ell_{\epsilon}$ such that $\Pi_{\ell_{\epsilon}}$ is an $\epsilon$-mapping; that is, for all $y \in X_{\ell_{\epsilon}}$ the inverse image set $\Pi_{\ell_{\epsilon}}^{-1}(y) \subset \mathfrak{M}$ has diameter less than $\epsilon$. The notion of an $\epsilon$-map was introduced in 1928 by Alexandroff [ 5 :

2010 Mathematics Subject Classification. Primary 52C23, 57R05, 54F15, 37B45; Secondary 53C12, 57N55 .

Version date: November 1, 2018 . 
DEFINITION 1.2. Let $\mathfrak{M}$ be a continuum, $Y$ a topological space and $\epsilon>0$ a constant. A map $f: \mathfrak{M} \rightarrow Y$ is said to be an $\epsilon$-map if $f$ is a continuous surjection, and for each point $y \in Y$, the inverse image $f^{-1}(y)$ has diameter less than $\epsilon$. A continuum $\mathfrak{M}$ is said to be $Y$-like, for some topological space $Y$, if for every $\epsilon>0$, there is an $\epsilon$-map $f_{\epsilon}: \mathfrak{M} \rightarrow Y$. More generally, let $\mathcal{Y}$ be a collection of compact topological spaces, then $\mathfrak{M}$ is $\mathcal{Y}$-like if for all $\epsilon>0$, there exists $Y_{\epsilon} \in \mathcal{Y}$ and an $\epsilon-$ map $f_{\epsilon}: \mathfrak{M} \rightarrow Y_{\epsilon}$.

In this work, we consider the following special case of the above definition:

DEFINITION 1.3. A continuum $\mathfrak{M}$ is manifold-like, if given any $\epsilon>0$, there exists a closed manifold $M_{\epsilon}$ and an $\epsilon$-map $f: \mathfrak{M} \rightarrow M_{\epsilon}$. For a closed manifold $M$, a continuum $\mathfrak{M}$ is $M$-like, if given any $\epsilon>0$, there exists an $\epsilon$-map $f: \mathfrak{M} \rightarrow M$.

The following is then a natural problem to investigate:

PROBLEM 1.4. For a closed $n$-manifold $M$, characterize the continua which are $M$-like.

Problem 1.4 is too broad to obtain definitive results, as there exist too many pathological constructions of $M$-like spaces. The simplest case, where $M=[0,1]$ is an interval, has been extensively studied for many special classes of bonding maps, such as the "tent maps", where many results are known for the inverse limit spaces. However, without some sort of "regularity" restriction, even the continua modeled on bonding maps to $[0,1]$ are too wild to hope to classify. Thus, one imposes additional assumptions on the continua considered, such as the following. Recall that a topological space $\mathfrak{M}$ is homogeneous if for every $x, y \in \mathfrak{M}$, there exists a homeomorphism $h: \mathfrak{M} \rightarrow \mathfrak{M}$ such that $h(x)=y$. A continuum $X$ is circle-like if it is $M$-like, where $M=\mathbb{S}^{1}$ is the circle. The following is a well-known result of R.H. Bing, which has inspired many generalizations:

THEOREM 1.5 (Bing [9]). Let $\mathfrak{M}$ be a homogeneous, circle-like continuum that contains an arc. Then either $\mathfrak{M}$ is homeomorphic to $\mathbb{S}^{1}$, or to an inverse limit of proper finite coverings of $\mathbb{S}^{1}$.

The hypothesis in Theorem 1.5 that $\mathfrak{M}$ is homogeneous and contains an arc implies that for every point $x \in \mathfrak{M}$, there is an arc containing $x$. One generalization of this hypothesis is suggested by the properties of continua arising from the investigation of the dynamical properties of smooth foliations of compact manifolds, for example as discussed in [47. In this case, the invariant continua have the structure of foliated spaces, in the sense of [61. That is, for each $x \in \mathfrak{M}$, there is a neighborhood $x \in U_{x} \subset \mathfrak{M}$ where $U_{x}$ is homeomorphic to a product of an open subset of $\mathbb{R}^{n}$, for some $n \geq 1$, with a closed subset $\mathfrak{T}_{x}$ of some Polish space. If the transversal spaces $\mathfrak{T}_{x}$ are totally disconnected for all $x \in \mathfrak{M}$, and are not singleton spaces, then we say that $\mathfrak{M}$ is a matchbox manifold, and $\mathfrak{M}$ then admits a decomposition $\mathcal{F}_{\mathfrak{M}}$ into path-connected components of constant dimension $n$ which are the leaves of $\mathcal{F}_{\mathfrak{M}}$. A matchbox manifold with 2-dimensional leaves is a lamination by surfaces in the sense of Ghys 43 and Lyubich and Minsky [55, while Sullivan called them "solenoidal spaces" in 174, 78. The terminology "matchbox manifold" follows the usage introduced in [1, 3, 4, and as used in the authors' works [15, 16, 17, 18, 19, 31, 32, 33, 48, 49] which study this class of continua.

In the authors' previous work 19 we have shown:

THEOREM 1.6. [19, Corollary 1.6] Let $\mathfrak{M}$ be a manifold-like matchbox manifold $\mathfrak{M}$. Then there exists a presentation $\mathcal{P}=\left\{p_{\ell+1}: M_{\ell+1} \rightarrow M_{\ell} \mid \ell \geq 0\right\}$, where each bonding map $p_{\ell+1}$ is a proper covering map, and a homeomorphism

$$
\Phi_{\mathcal{P}}: \mathfrak{M} \cong \mathcal{S}_{\mathcal{P}} \equiv \lim _{\longleftarrow}\left\{p_{\ell+1}: M_{\ell+1} \rightarrow M_{\ell} \mid \ell \geq 0\right\} .
$$

A continuum given by the inverse limit of a sequence of proper covering maps is called a weak solenoid, following the usage in the the works of Rogers and Tollefson [66, 67, 68, 69. The properties of these spaces are discussed further in Section 2 ,

Note that a homeomorphism $\Phi_{\mathcal{P}}: \mathfrak{M} \rightarrow \mathcal{S}_{\mathcal{P}}$ preserves path components, hence maps the leaves of the foliation $\mathcal{F}_{\mathfrak{M}}$ homeomorphically onto the leaves of the foliation $\mathcal{F}_{\mathcal{P}}$ of $\mathcal{S}_{\mathcal{P}}$. 
Mardesic and Segal discuss pointed shape expansions in [57, and show there is well-defined notion of pro-homotopy groups pro- $\pi_{k}(\mathfrak{M}, z)$ of a space $\mathfrak{M}$ using pointed shape exapnsions, though it may depend on the choice of basepoint $z \in \mathfrak{M}$. For example, see Example 4, [57, Chapter II, Section 3.3]. One of the main technical points of this work, is that the pro-homotopy groups are well-defined for a weak solenoid, independent of the choice of basepoint $z \in \mathfrak{M}$.

Here is our first result, which is a general property of weak solenoids, and can be considered a formal version of the ideas of Fokkink and Oversteegen in 38 .

THEOREM 1.7. Let $\mathfrak{M}$ be an $M$-like matchbox manifold, then the pro-group pro- $\pi_{1}(\mathfrak{M}, z)$ is independent of the choice of basepoint $z \in \mathfrak{M}$ up to isomorphism, and depends only on the homeomorphism class of $\mathfrak{M}$.

Pro-groups are discussed in Section 4 , then the definition of pro- $\pi_{1}(\mathfrak{M}, z)$ is given in Section 5 and we show that it is well-defined. Then in Section [6, we calculate the pro-group associated to an $M$-like matchbox manifold, which leads to the following generalization of Theorem 1.5 by Bing:

THEOREM 1.8. Let $\mathfrak{M}$ be an n-dimensional matchbox manifold which is $M$-like, for a closed manifold $M$. Then $\mathfrak{M}$ admits a presentation $\mathcal{P}=\left\{q_{\ell+1}: N_{\ell+1} \rightarrow N_{\ell} \mid \ell \geq 0\right\}$, where each $N_{\ell}$ is a closed n-manifold whose fundamental group is isomorphic to that of $M$, and each $q_{\ell+1}: N_{\ell+1} \rightarrow N_{\ell}$ is a proper covering map for $\ell \geq 0$, such that there is a homeomorphism

$$
\Phi_{\mathcal{P}}: \mathfrak{M} \cong \mathcal{S}_{\mathcal{P}} \equiv \lim _{\longleftarrow}\left\{q_{\ell+1}: N_{\ell+1} \rightarrow N_{\ell} \mid \ell \geq 0\right\} .
$$

The conclusion of Theorem 1.8 can be strengthened if we impose additional hypotheses on the target space $M$. Recall that a finite $C W$-complex $Y$ is aspherical if it is connected and its universal covering space is contractible. The Borel Conjecture is that if $Y_{1}$ and $Y_{2}$ are homotopy equivalent, aspherical closed manifolds, then a homotopy equivalence between $Y_{1}$ and $Y_{2}$ is homotopic to a homeomorphism between $Y_{1}$ and $Y_{2}$. The Borel Conjecture has been proven for many classes of aspherical manifolds:

(1) the torus $\mathbb{T}^{n}$ for all $n \geq 1$,

(2) all infra-nilmanifolds,

(3) closed Riemannian manifolds $Y$ with negative sectional curvatures,

(4) closed Riemannian manifolds $Y$ of dimension $n \neq 3$, 4 with non-positive sectional curvatures.

The above list is not exhaustive, and only cases (1) and (2) are used in this paper. The history and status of the Borel Conjecture is discussed, for example, in the surveys of Davis [21] and Lück [52.

A manifold for which all finite coverings satisfy the Borel Conjecture was called strongly Borel in the authors work [18. Note that the class of "D-like" spaces introduced in [70, Section 5] consists of manifolds which are strongly Borel. Here is our second main result, which is a further generalization of Theorem 1.5 by Bing:

THEOREM 1.9. Let $M$ be a closed aspherical n-manifold $M$ which satisfies the Borel Conjecture. If $\mathfrak{M}$ is a matchbox manifold which is $M$-like, then $\mathfrak{M}$ admits a presentation $\mathcal{P}=\left\{q_{\ell+1}: M \rightarrow\right.$ $M \mid \ell \geq 0\}$, where each $q_{\ell+1}: M \rightarrow M$ is a proper covering map for $\ell \geq 0$, such that there is a homeomorphism

$$
\Phi_{\mathcal{P}}: \mathfrak{M} \cong \lim _{\longleftarrow}\left\{q_{\ell+1}: M \rightarrow M \mid \ell \geq 0\right\}
$$

As an example, note that the $n$-torus $\mathbb{T}^{n}$ satisfies the hypotheses of Theorem 1.9, so we conclude:

COROLLARY 1.10. Let $\mathfrak{M}$ be a matchbox manifold which is $\mathbb{T}^{n}$-like, then it admits a presentation as in (5) for $M=\mathbb{T}^{n}$.

Recall that a group $H$ is said to be (finitely) non co-Hopfian, or just non co-Hopfian in this work, if there is an injective homomorphism $\iota: H \rightarrow H$ whose image has finite index, but is not onto. That is, $H$ contains a subgroup of finite index isomorphic to itself. The class of non co-Hopfian groups has been extensively studied, as discussed further in Section 8 , providing many examples. The general philosophy is that the group $H$ should be "essentially" nilpotent. A complete solution of 
Problem 1.4 in the case of matchbox manifolds then requires a classification of the finitely-generated, non co-Hopfian groups. The works of van Limbeek [76, 77, give some indications of the available techniques for the study of this problem, as well as some of the difficulties it presents.

The proofs of Theorems 1.8 and 1.9 are given in Section 7 . Finally, Section 8 gives collections of examples of weak solenoids and non co-Hopfian groups illustrating the conclusions of theorems, and showing that stronger results are not possible without further assumptions.

\section{WEAK SOLENOIDS}

A weak solenoid $\mathcal{S}_{\mathcal{P}}$ of dimension $n \geq 1$, is the inverse limit space of a sequence

$$
\begin{aligned}
\mathcal{S}_{\mathcal{P}} & \equiv \lim _{\longleftarrow}\left\{p_{\ell+1}: M_{\ell+1} \rightarrow M_{\ell}\right\} \\
& =\left\{\left(x_{0}, x_{1}, \ldots\right) \in \mathcal{S}_{\mathcal{P}} \mid p_{\ell+1}\left(x_{\ell+1}\right)=x_{\ell} \text { for all } \ell \geq 0\right\} \subset \prod_{\ell \geq 0} M_{\ell}
\end{aligned}
$$

where each $M_{\ell}$ is a compact connected $n$-dimensional manifold without boundary, and each $p_{\ell+1}$ is a covering map of finite degree greater than one. The set $\mathcal{S}_{\mathcal{P}}$ is given the relative topology, induced from the product (Tychonoff) topology, so that $\mathcal{S}_{\mathcal{P}}$ is itself compact and connected. The collection

$$
\mathcal{P}=\left\{p_{\ell+1}: M_{\ell+1} \rightarrow M_{\ell} \mid \ell \geq 0\right\},
$$

is called a presentation for $\mathcal{S}_{\mathcal{P}}$. For example, a Vietoris solenoid [75, 79] is a 1-dimensional solenoid, where each $M_{\ell}$ is a circle, as arises in the conclusion of Theorem 1.5

Let $\mathcal{S}_{\mathcal{P}}$ be a weak solenoid with presentation $\mathcal{P}$ as in (7). For each $\ell \geq 1$, the composition

$$
p_{\ell}^{0}=p_{1} \circ \cdots \circ p_{\ell-1} \circ p_{\ell}: M_{\ell} \rightarrow M_{0}
$$

is a finite-to-one covering map of the base manifold $M_{0}$. For each $\ell \geq 0$, projection onto the $\ell$-th factor in the product $\prod_{\ell \geq 0} M_{\ell}$ in (6) yields a surjective map denoted by $\Pi_{\ell}^{\mathcal{P}}: \mathcal{S}_{\mathcal{P}} \rightarrow M_{\ell}$. Then for $\ell \geq 1$, we have $\Pi_{0}^{\mathcal{P}}=p_{\ell}^{0} \circ \Pi_{\ell}^{\mathcal{P}}: \mathcal{S}_{\mathcal{P}} \rightarrow M_{0}$.

McCord showed in [59] that for each $\ell>0$, the map $\Pi_{\ell}^{\mathcal{P}}: \mathcal{S}_{\mathcal{P}} \rightarrow M_{\ell}$ is a fibration over $M_{\ell}$. For a choice of a basepoint $x_{0} \in M_{0}$, the assumption that the fibers of each map $p_{\ell+1}$ have cardinality at least 2 implies the fiber $\mathfrak{X}_{0}=\left(\Pi_{0}^{\mathcal{P}}\right)^{-1}\left(x_{0}\right)$ is a Cantor set. McCord also observed that $\mathcal{S}_{\mathcal{P}}$ has a local product structure, which implies that the path-connected components of $\mathcal{S}_{\mathcal{P}}$ define a foliation denoted by $\mathcal{F}_{\mathcal{P}}$. That is, $n$-dimensional weak solenoids are examples of $n$-dimensional matchbox manifolds. The properties of weak solenoids have been studied in the works by Schori [72, Rogers and Tollefson [66, 67, 68, 69, Fokkink and Oversteegen in [38, and by the authors.

Given a basepoint $x \in \mathcal{S}_{\mathcal{P}}$ for each $\ell \geq 0$ we then obtain basepoints $x_{\ell}=\Pi_{\ell}(x) \in M_{\ell}$. Denote by $G_{0}=\pi_{1}\left(M_{0}, x_{0}\right)$ the fundamental group of $M_{0}$ with basepoint $x_{0}$, and for $\ell>0$ let

$$
G_{\ell}=\text { image }\left\{p_{\ell}^{0}: \pi_{1}\left(M_{\ell}, x_{\ell}\right) \longrightarrow G_{0}\right\}
$$

where by a small abuse of notation, we let $p_{\ell}^{0}$ also denote the induced map on fundamental groups, and also suppress the dependence on the choice of basepoint $x$ in the notation $G_{\ell}$. Note that each $G_{\ell}$ is a subgroup of finite index in $G_{0}$. In this way, associated to the presentation $\mathcal{P}$ and basepoint $x \in \mathfrak{X}_{0}$, we obtain a descending chain of subgroups of finite index

$$
\mathcal{G}_{x} \equiv\left\{G_{0} \supset G_{1} \supset G_{2} \supset \cdots \supset G_{\ell} \supset \cdots\right\} .
$$

Each quotient $X_{\ell}=G_{0} / G_{\ell}$ is a finite set equipped with a left $G_{0}$-action, and there are surjections $X_{\ell+1} \rightarrow X_{\ell}$ which commute with the action of $G_{0}$. The inverse limit,

$$
X(\mathcal{G})=\lim _{\longleftarrow}\left\{p_{\ell+1}: X_{\ell+1} \rightarrow X_{\ell} \mid \ell \geq 0\right\}=\left\{\left(e G_{0}, g_{1} G_{1}, \ldots\right) \mid g_{\ell} G_{\ell}=g_{\ell+1} G_{\ell}\right\} \subset \prod_{\ell \geq 0} X_{\ell}
$$

is then a totally-disconnected, compact, perfect set for the topology induced from the Tychonoff topology on the product, so is a Cantor set. Note that there is a canonical basepoint $\left(e G_{\ell}\right) \in X(\mathcal{G})$ where $e \in G_{0}$ is the identity element of the group. The fundamental group $G_{0}$ acts on the left on 
$X(\mathcal{G})$ via the coordinate-wise multiplication on the product in (11). The left action of $G_{0}$ on each quotient space $X_{\ell}$ is transitive, so each orbit of $G_{0}$ acting on $X(\mathcal{G})$ is dense. We denote this minimal Cantor action by $\Phi_{0}: G_{0} \times X(\mathcal{G}) \rightarrow X(\mathcal{G})$, or by $\left(X(\mathcal{G}), G_{0}, \Phi_{0}\right)$.

The space $X(\mathcal{G})$ admits a natural identification with the fiber $\mathfrak{X}_{0} \equiv \Pi_{0}^{-1}\left(x_{0}\right)$. The Cantor action $\left(X(\mathcal{G}), G_{0}, \Phi_{0}\right)$ is then conjugate with the monodromy action of $G_{0}$ on $\mathfrak{X}_{0}$ induced by the leaves of the foliation $\mathcal{F}_{\mathcal{P}}$. See [33] for more details on this identification, and also the dependence on the choice of the basepoint $x \in \mathfrak{X}_{0}$.

A group chain $\mathcal{G}$ as in (10) determines a presentation $\mathcal{P}$ with pointed base manifold $\left(M_{0}, x_{0}\right)$, and thus a weak solenoid $\mathcal{S}_{\mathcal{P}}$. We next consider two equivalence relations on group chains, which were introduced by Rogers and Tollefson in [68] and Fokkink and Oversteegen in 38 .

Let $\mathfrak{G}$ denote the collection of all subgroup chains in $G_{0}$. The first notion of equivalence for elements of $\mathfrak{G}$ corresponds to the standard notion of intertwined chains:

DEFINITION 2.1. (Rogers-Tollefson, [68]) Group chains $\mathcal{G}=\left\{G_{\ell}\right\}_{\ell \geq 0}$ and $\mathcal{H}=\left\{H_{\ell}\right\}_{\ell \geq 0}$ are equivalent if $G_{0}=H_{0}$ and there is a group chain $\mathcal{K}=\left\{K_{\ell}\right\}_{\ell \geq 0}$ with $K_{0}=G_{0}$, and infinite subsequences $\left\{G_{\ell_{k}}\right\}_{k \geq 0}$ and $\left\{H_{j_{k}}\right\}_{k \geq 0}$ such that $K_{2 k}=G_{\ell_{k}}$ and $K_{2 k+1}=H_{j_{k}}$ for $k \geq 0$.

The second notion of equivalence is more subtle, as it corresponds to conjugating a group chain by an element of the profinite core group $C_{\infty}$ as discussed in [31].

DEFINITION 2.2. (Fokkink-Oversteegen, 38) Group chains $\mathcal{G}=\left\{G_{\ell}\right\}_{\ell \geq 0}$ and $\mathcal{H}=\left\{H_{\ell}\right\}_{\ell \geq 0}$ in $\mathfrak{G}$ are conjugate equivalent if $G_{0}=H_{0}$, and:

(1) there exists a sequence $\left(g_{\ell}\right)_{\ell \geq 0}$ where each $g_{\ell} \in G_{0}$;

(2) the compatibility condition $g_{\ell} G_{\ell}=g_{\ell+1} G_{\ell}$ for all $\ell \geq 0$ is satisfied;

(3) the group chains $\mathcal{G}^{\left(g_{\ell}\right)}=\left\{g_{\ell} G_{\ell} g_{\ell}^{-1}\right\}_{\ell \geq 0}$ and $\mathcal{H}=\left\{\bar{H}_{\ell}\right\}_{\ell \geq 0}$ are equivalent.

The dynamical meaning of the equivalences in Definitions 2.1 and 2.2 is given by the following theorem, which follows from results in [38; ; see also [31.

THEOREM 2.3. Let $\mathcal{G}=\left\{G_{\ell}\right\}_{\ell \geq 0}$ and $\mathcal{H}=\left\{H_{\ell}\right\}_{\ell \geq 0}$ be group chains with $H_{0}=G_{0}$, and let

$$
\begin{aligned}
& X(\mathcal{G})=\lim \left\{G_{0} / G_{\ell+1} \rightarrow G_{0} / G_{\ell}\right\}, \\
& X(\mathcal{H})=\lim _{\leftarrow}\left\{H_{0} / H_{\ell+1} \rightarrow H_{0} / H_{\ell}\right\} .
\end{aligned}
$$

Then:

(1) The group chains $\mathcal{G}$ and $\mathcal{H}$ are equivalent if and only if there exists a homeomorphism $\tau: X(\mathcal{G}) \rightarrow X(\mathcal{H})$ equivariant with respect to the $G_{0}$-actions on $X(\mathcal{G})$ and $X(\mathcal{H})$, and such that $\phi\left(e G_{\ell}\right)=\left(e H_{\ell}\right)$.

(2) The group chains $\mathcal{G}$ and $\mathcal{H}$ are conjugate equivalent if and only if there exists a homeomorphism $\tau: X(\mathcal{G}) \rightarrow X(\mathcal{H})$ equivariant with respect to the $G_{0}$-actions on $X(\mathcal{G})$ and $X(\mathcal{H})$.

That is, an equivalence of two group chains corresponds to the existence of a basepoint-preserving equivariant homeomorphism between their inverse limit systems, while a conjugate equivalence of two group chains corresponds to the existence of a equivariant conjugacy between their inverse limit systems, which need not preserve the basepoint.

REMARK 2.4. The kernel of a group chain $\mathcal{G}=\left\{G_{\ell}\right\}_{\ell \geq 0}$ is the subgroup of $G_{0}$ given by

$$
K(\mathcal{G})=\bigcap_{\ell \geq 0} G_{\ell}
$$

It follows immediately from the definitions that if the group chains $\mathcal{G}=\left\{G_{\ell}\right\}_{\ell \geq 0}$ and $\mathcal{H}=\left\{H_{\ell}\right\}_{\ell \geq 0}$ with $G_{0}=H_{0}$ are equivalent, then $K(\mathcal{G})=K(\mathcal{H}) \subset G_{0}$. If the chains $\mathcal{G}$ and $\mathcal{H}$ are only conjugate equivalent, then the kernels need not be equal. Thus, the kernel $K(\mathcal{G})$ of a group chain $\mathcal{G}$ is an invariant of equivalence, but is not necessarily invariant by conjugate equivalence. 
The kernel $K(\mathcal{G})$ has an interpretation in terms of the topology of the leaves of the foliation $\mathcal{F}_{\mathcal{P}}$ of the weak solenoid $\mathcal{S}_{\mathcal{P}}$. Choose a basepoint $x \in \Pi_{0}^{-1}\left(x_{0}\right)$ and let $L_{x} \subset \mathcal{S}_{\mathcal{P}}$ be the leaf containing $x$. The restriction of the bundle projection $\left.\Pi_{0}\right|_{L_{x}}: L_{x} \rightarrow M_{0}$ is a covering map. Let $\widetilde{M}_{0}$ be the universal cover of $M_{0}$. Then by standard arguments of covering space theory (see also McCord [59]) there is a homeomorphism $\widetilde{M}_{0} / K(\mathcal{G}) \rightarrow L_{x}$. In particular, if $L_{x}$ is simply connected, then $K(\mathcal{G})=\{e\}$.

A standard fact about weak solenoids, is that their homeomorphism type remains unchanged if a finite number of the initial maps in a presentation $\mathcal{P}$ as in (7) are deleted. See [18, 33, 49] for discussions of this property and its significance. This fact suggests a third type of equivalence relation on group chains. For a group chain $\mathcal{G}=\left\{G_{\ell}\right\}_{\ell \geq 0}$ and $k \geq 0$, define the "truncated group chain" $\mathcal{G}^{(k)}$ by setting where $G_{\ell}^{(k)}=G_{\ell+k}$, then

$$
\mathcal{G}^{(k)}: G_{0}^{(k)} \supset G_{1}^{(k)} \supset G_{2}^{(k)} \supset \cdots \supset G_{\ell}^{(k)} \supset \cdots .
$$

DEFINITION 2.5. Group chains $\mathcal{G}=\left\{G_{\ell}\right\}_{\ell \geq 0}$ and $\mathcal{H}=\left\{H_{\ell}\right\}_{\ell \geq 0}$ are return equivalent if there exists integers $k, m \geq 0$ such that the truncated group chains $\mathcal{G}^{(k)}$ and $\mathcal{H}^{(m)}$ are equivalent in the sense of Definition 2.1. We say they are conjugate return equivalent if there exists integers $k, m \geq 0$ such that the truncated group chains $\mathcal{G}^{(k)}$ and $\mathcal{H}^{(m)}$ are equivalent in the sense of Definition 2.2.

Recall that for the Tychonoff topology on $X(\mathcal{G})$, we have a descending chain $\left\{U_{k}(\mathcal{G}) \mid k=0,1,2, \ldots\right\}$ of clopen neighborhoods of the basepoint $\left(e G_{\ell}\right)$, where

$$
U_{k}(\mathcal{G})=\lim _{\longleftarrow}\left\{p_{\ell+1}: X_{\ell+1} \rightarrow X_{\ell} \mid \ell \geq k\right\}
$$

The spaces $U_{k}(\mathcal{G})$ and $X\left(\mathcal{G}^{(k)}\right)$ are canonically isomorphic, and the action of $G_{k}$ on $X\left(\mathcal{G}^{(k)}\right)$ is conjugate to the restricted action of $G_{k}$ on $U_{k}(\mathcal{G})$. Thus, in the terminology of [49] we have:

PROPOSITION 2.6. Given group chains $\mathcal{G}=\left\{G_{\ell}\right\}_{\ell \geq 0}$ and $\mathcal{H}=\left\{H_{\ell}\right\}_{\ell \geq 0}$, then:

(1) $\mathcal{G}$ and $\mathcal{H}$ are return equivalent if an only if the minimal Cantor actions $\left(X(\mathcal{G}), G_{0}, \Phi_{0}\right)$ and $\left(X(\mathcal{H}), H_{0}, \Psi_{0}\right)$ are return equivalent by a basepoint preserving local homeomorphism.

(2) $\mathcal{G}$ and $\mathcal{H}$ are conjugate return equivalent if and only if the minimal Cantor actions $\left(X(\mathcal{G}), G_{0}, \Phi_{0}\right)$ and $\left(X(\mathcal{H}), H_{0}, \Psi_{0}\right)$ are return equivalent.

Finally, we recall a basic result from [18] that was mentioned above as the motivation for introducing the equivalence relation in Definition 2.5.

THEOREM 2.7. Let $\mathcal{S}_{\mathcal{P}}$ and $\mathcal{S}_{\mathcal{P}^{\prime}}$ be weak solenoids associated to presentations $\mathcal{P}$ and $\mathcal{P}^{\prime}$ with a common base manifolds, $M_{0}=M_{0}^{\prime}$. Let $\mathcal{G}$ be the group chain associated to $\mathcal{P}$, and $\mathcal{G}^{\prime}$ be the group chain associated to $\mathcal{P}^{\prime}$. If $\mathcal{S}_{\mathcal{P}}$ and $\mathcal{S}_{\mathcal{P}^{\prime}}$ are homeomorphic as continua, then the group chains $\mathcal{G}$ and $\mathcal{G}^{\prime}$ are conjugate return equivalent.

There is a converse to this result, which requires additional assumptions on the base manifold $M_{0}$ and the geometry of the foliations, as given in [18, Theorem 1.5]. The conclusion of Theorem 2.7 is one of the motivations for the study of return equivalence of group chains, with the goal of obtaining computable invariants. The asymptotic discriminant, introduced in [48, and further studied in [49], is an invariant of conjugate return equivalence. The growth of orbits as discussed in [32] is another such invariant.

\section{INDUCED MAPS BETWEEN CONTINUA}

We recall some results from the literature, concerning the properties of induced maps between continua as defined by maps between their presentations as in (2). The notion of equivalence is a fundamental aspect of the theory of shape equivalence, as developed for example in [57. In order to apply these results to the group chains associated to presentations, care must be taken with respect to basepoints. For this reason, we take our definitions from the original works by Bousfield and Kan [12, Fort and McCord [39, Mioduszewski [60, and Rogers and Tollefson [67, 68. These results are then applied for the presentations associated to a matchbox manifold which is $M$-like. 
Assume there are given presentations

$$
\begin{aligned}
& \mathcal{P}=\left\{f_{\ell+1}: X_{\ell+1} \rightarrow X_{\ell} \mid \ell \geq 0\right\} \\
& \mathcal{Q}=\left\{g_{\ell+1}: Y_{\ell+1} \rightarrow Y_{\ell} \mid \ell \geq 0\right\},
\end{aligned}
$$

where for all $\ell \geq 0$, the spaces $X_{\ell}$ and $Y_{\ell}$ are compact connected polyhedra, and the maps $f_{\ell+1}$ and $g_{\ell+1}$ are continuous surjections. Let $\mathcal{S}_{\mathcal{P}}$ be the inverse limit defined by $\mathcal{P}$, and $\mathcal{S}_{\mathcal{Q}}$ the inverse limit of $\mathcal{Q}$. A pro-map from $\mathcal{P}$ to $\mathcal{Q}$ is a collection $\widehat{\sigma} \equiv\left\{\sigma_{\ell}: X_{m_{\ell}} \rightarrow Y_{\ell} \mid \ell \geq 0\right\}$, where

(1) $0 \leq m_{0}<m_{1}<m_{2}<\cdots$ is an increasing sequence,

(2) $\sigma_{\ell}: X_{m_{\ell}} \rightarrow Y_{\ell}$ are continuous maps for $\ell \geq 0$,

and moreover, for each $\ell>0$ and $f_{m_{\ell}}^{m_{\ell+1}}=f_{m_{\ell}+1} \circ \cdots \circ f_{m_{\ell+1}}$, the following diagram commutes

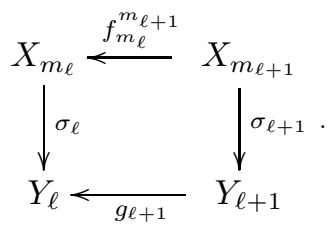

A pro-map $\widehat{\sigma}$ determines a continuous map $S(\widehat{\sigma}): \mathcal{S}_{\mathcal{P}} \rightarrow \mathcal{S}_{\mathcal{Q}}$ called the induced map for $\widehat{\sigma}$.

Recall that $\Pi_{\ell}^{\mathcal{P}}: \mathcal{S}_{\mathcal{P}} \rightarrow X_{\ell}$ and $\Pi_{\ell}^{\mathcal{Q}}: \mathcal{S}_{\mathcal{Q}} \rightarrow Y_{\ell}$ denote the projections on the factors of these spaces. Then the defining property for an induced map is that for all $\ell>0$, we have

$$
\Pi_{\ell}^{\mathcal{Q}} \circ S(\widehat{\sigma})=\sigma_{\ell} \circ \Pi_{m_{\ell}}^{\mathcal{P}}: \mathcal{S}_{\mathcal{P}} \rightarrow Y_{\ell} .
$$

A fundamental question is when is there an induced map $S(\widehat{\sigma})$ which equals $h$, or is approximately equal to $h$ in an appropriate sense? This problem has been analyzed in the works [60, 39, 51, 66, 67, 70, 28, 29, and we recall their conclusions as needed for the study of $M$-like spaces.

The first result was obtained by Mioduszewski in [60], where he introduced a generalization of the notion of a map between resolutions, where the hypothesis that the diagram (17) commutes is replaced by a condition that it almost commutes. To be more precise, first assume that each space $Y_{\ell}$ has a metric $d_{Y_{\ell}}$ defining its topology, for $\ell \geq 0$. Let $D\left(Y_{\ell}\right)>0$ be the diameter of $Y_{\ell}$ for this metric, then and give the inverse limit $\mathcal{S}_{\mathcal{Q}}$ the metric

$$
\left.\left.d_{\mathcal{S}_{\mathcal{Q}}}\left(\left(y_{\ell}\right)_{\ell \geq 0}\right),\left(y_{\ell}^{\prime}\right)_{\ell \geq 0}\right)\right)=\sum_{\ell \geq 0} \frac{1}{2^{-\ell} D\left(Y_{\ell}\right)} \cdot d_{Y_{\ell}}\left(y_{\ell}, y_{\ell}^{\prime}\right) .
$$

Assume that there is given a descending sequence $\widehat{\epsilon} \equiv\left\{\epsilon_{0}>\epsilon_{1}>\epsilon_{2}>\cdots\right\}$ with $\lim _{\ell \rightarrow \infty} \epsilon_{\ell}=0$. Then a sequence of maps $\widehat{\sigma}$ as above is said to be an $\widehat{\epsilon}$-pro-map if, in place of the assumption that the diagrams (17) are commutative, we assume that for all $\ell>0$,

$$
d_{Y_{\ell}}\left(g_{\ell+1} \circ \sigma_{\ell+1}(x), \sigma_{\ell} \circ f_{m_{\ell}}^{m_{\ell+1}}(x)\right)<\epsilon_{\ell} \quad \text { for all } x \in X_{m_{\ell+1}} .
$$

By [60, Theorem 2'], an $\widehat{\epsilon}$-pro-map $\widehat{\sigma}$ determines a continuous map $S(\widehat{\sigma}): \mathcal{S}_{\mathcal{P}} \rightarrow \mathcal{S}_{\mathcal{Q}}$. Moreover, by the results of [60, Section 3], if there is given a homeomorphism $\Phi: \mathcal{S}_{\mathcal{P}} \rightarrow \mathcal{S}_{\mathcal{Q}}$ then there exists $\widehat{\epsilon}$ as above, and an $\widehat{\epsilon}$-pro-map $\widehat{\sigma}$ such that the induced map $S(\widehat{\sigma}): \mathcal{S}_{\mathcal{P}} \rightarrow \mathcal{S}_{\mathcal{Q}}$ is a homeomorphism. A natural problem is to then ask when the induced map $S(\widehat{\sigma})$ is equal to the map $\Phi$. Fort and McCord gave a partial answer to this question, in the case where the target space $\mathcal{S}_{\mathcal{Q}}$ is a weak solenoid:

THEOREM 3.1. 39, Theorem 1] Let $\mathcal{P}$ be a presentation whose spaces $X_{\ell}$ are compact connected polyhedra, and let $\mathcal{Q}$ be a presentation consisting of covering maps. Assume that all spaces have metrics as above. Then for any map $\Phi: \mathcal{S}_{\mathcal{P}} \rightarrow \mathcal{S}_{\mathcal{Q}}$ and $\epsilon>0$, there exists a descending sequence $\widehat{\epsilon}$ as above, and an $\widehat{\epsilon}$-pro-map $\widehat{\sigma}$ such that the induced map $S(\widehat{\sigma}): \mathcal{S}_{\mathcal{P}} \rightarrow \mathcal{S}_{\mathcal{Q}}$ is $\epsilon$-homotopic to $\Phi$. In particular, the maps $\Phi$ and $S(\widehat{\sigma})$ are $\epsilon$-close.

In the proof of this result, the fact that each bonding map $q_{\ell}$ is a covering is used to lift a homotopy from the approximation at level $\ell$ to one at level $\ell+1$ (see the proof of [39, Lemma 5]). Rogers and Tollefson gave a further refinement of Theorem [3.1] in [67, characterizing the maps for which one 
can obtain $h=S(\widehat{\sigma})$ in terms of whether $h$ is locally fiber preserving, and in [70] they applied their method to the case where both presentations consists of covering maps.

Next, let $\mathfrak{M}$ be a matchbox manifold which is $M$-like, where $M$ is a closed manifold. Then by Theorem 1.6. there exists a presentation $\mathcal{P}=\left\{p_{\ell+1}: M_{\ell+1} \rightarrow M_{\ell} \mid \ell \geq 0\right\}$ where each bonding map $p_{\ell+1}$ is a proper covering map, and a homeomorphism

$$
\Phi_{\mathcal{P}}: \mathfrak{M} \cong \mathcal{S}_{\mathcal{P}} \equiv \lim _{\longleftarrow}\left\{p_{\ell+1}: M_{\ell+1} \rightarrow M_{\ell} \mid \ell \geq 0\right\} .
$$

The choice of such a presentation is not unique. The result of Rogers and Tollefson [70, Theorem 4.6] relates two such presentations.

THEOREM 3.2. Suppose there are two presentations as weak solenoids

$$
\begin{aligned}
& \mathcal{P}=\left\{p_{\ell+1}: M_{\ell+1} \rightarrow M_{\ell} \mid \ell \geq 0\right\} \\
& \mathcal{Q}=\left\{q_{\ell+1}: N_{\ell+1} \rightarrow N_{\ell} \mid \ell \geq 0\right\},
\end{aligned}
$$

a homeomorphism $\Phi: \mathcal{S}_{\mathcal{P}} \rightarrow \mathcal{S}_{\mathcal{Q}}$ and $\epsilon>0$. Then there exists a decreasing sequence $\widehat{\epsilon}$ with $\epsilon_{0} \leq \epsilon$,

(1) an intertwined increasing sequence $0 \leq j_{0}<i_{0}<j_{1}<i_{1}<j_{2}<i_{2}<\cdots$,

(2) covering maps $\lambda_{\ell}: M_{i_{\ell+1}} \rightarrow N_{j_{\ell}}$ for $\ell \geq 0$,

(3) covering maps $\mu_{\ell}: N_{j_{\ell}} \rightarrow M_{i_{\ell}}$ for $\ell \geq 0$,

such that the following diagram $\widehat{\epsilon}$-commutes:

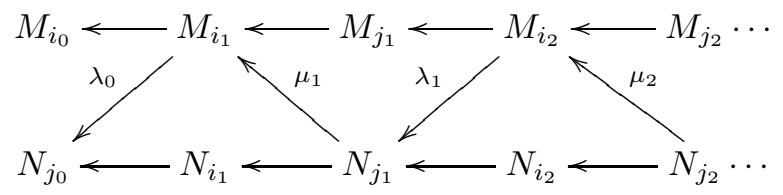

Moreover, for $\widehat{\lambda}=\left\{\lambda_{\ell}: M_{i_{\ell+1}} \rightarrow N_{j_{\ell}} \mid \ell \geq 0\right\}$, the induced map $S(\widehat{\lambda})$ is $\epsilon$-homotopic to $\Phi$, and similarly the induced map $S(\widehat{\mu})$ is $\epsilon$-homotopic to $\Phi^{-1}$.

We next recall an observation from Fokkink and Oversteegen in [38, based on 67, Theorem 2]. Let $\mathcal{P}$ and $\mathcal{Q}$ be presentations for weak solenoids and $\Phi: \mathcal{S}_{\mathcal{P}} \rightarrow \mathcal{S}_{\mathcal{Q}}$ a homeomorphism, as in Theorem 3.2 Choose a basepoint $x \in \mathcal{S}_{\mathcal{P}}$ and set $y=\Phi(x) \in \mathcal{S}_{\mathcal{Q}}$.

For $\ell \geq 0$, let $\Pi_{\ell}^{\mathcal{P}}: \mathcal{S}_{\mathcal{P}} \rightarrow M_{\ell}$ be the projection map onto the factor space $M_{\ell}$, and set $x_{\ell}=\Pi_{\ell}^{\mathcal{P}}(x) \in$ $M_{\ell}$. Then $X(\mathcal{P})_{\ell}=\left(\Pi_{\ell}^{\mathcal{P}}\right)^{-1}\left(x_{\ell}\right) \subset \mathcal{S}_{\mathcal{P}}$. Similarly, let $\Pi_{\ell}^{\mathcal{Q}}: \mathcal{S}_{\mathcal{Q}} \rightarrow N_{\ell}$ be the projection map onto the factor space $N_{\ell}$, and set $y_{\ell}=\Pi_{\ell}^{\mathcal{Q}}(y) \in N_{\ell}$. Then $X(\mathcal{Q})_{\ell}=\left(\Pi_{\ell}^{\mathcal{Q}}\right)^{-1}\left(y_{\ell}\right) \subset \mathcal{S}_{\mathcal{Q}}$.

REMARK 3.3. [38, Lemma 21] In the conclusion of Theorem 3.2, for $j_{0} \geq 0$ and $i_{1}>j_{0}$ sufficiently large, we can choose $\widehat{\lambda}$ so that the induced map satisfies $S(\widehat{\lambda})\left(X(\mathcal{P})_{i_{1}}\right)=X(\mathcal{Q})_{j_{0}}$.

Fokkink and Oversteegen use this remark in 38 to show that, in essence, associated to a weak solenoid $\mathfrak{M}$, there are well-defined pro-fundamental groups. This shown in Theorem 5.1 below.

Next, we recall the following sharper version of Theorem [1.1, due to Mardešić and Segal:

THEOREM 3.4. [56, Theorem $1^{*}$ ] Let $\mathfrak{M}$ be a continuum which is $M$-like for some closed connected manifold $M$. Then there exists a presentation $\mathcal{Q}=\left\{q_{\ell+1}: M \rightarrow M \mid \ell \geq 0\right\}$, where each map $q_{\ell+1}$ is a continuous surjection, and a homeomorphism $\Phi_{\mathcal{Q}}: \mathfrak{M} \cong \mathcal{S}_{\mathcal{Q}}$.

For an $M$-like matchbox manifold $\mathfrak{M}$, there is a presentation $\mathcal{P}$ as a weak solenoid and a homeomorphism $\Phi_{\mathcal{P}}: \mathfrak{M} \rightarrow \mathcal{S}_{\mathcal{P}}$, as in (21), and a presentation $\mathcal{Q}$ and a homeomorphism $\Phi_{\mathcal{Q}}: \mathfrak{M} \rightarrow \mathcal{S}_{\mathcal{Q}}$, as in Theorem 3.4. Set $\Phi=\mathcal{S}_{\mathcal{Q}} \circ \mathcal{S}_{\mathcal{P}}^{-1}: \mathcal{S}_{\mathcal{P}} \rightarrow \mathcal{S}_{\mathcal{Q}}$. Combining the results of this section, we then have:

THEOREM 3.5. Suppose there are presentations

$$
\begin{aligned}
& \mathcal{P}=\left\{p_{\ell+1}: M_{\ell+1} \rightarrow M_{\ell} \mid \ell \geq 0\right\} \\
& \mathcal{Q}=\left\{q_{\ell+1}: M \rightarrow M \mid \ell \geq 0\right\}
\end{aligned}
$$


where for $\ell \geq 0$, each $p_{\ell+1}$ is a covering map, each $q_{\ell+1}$ is a continuous surjection, and there is a homeomorphism $\Phi: \mathcal{S}_{\mathcal{P}} \rightarrow \mathcal{S}_{\mathcal{Q}}$. Assume that the presentations have metrics as in (19). Then for $\epsilon>0$ there exists a descending sequence $\widehat{\epsilon}$ with $0<\epsilon_{0}<\epsilon$ and:

(1) intertwined increasing sequence $0 \leq j_{0}<i_{0}<j_{1}<i_{1}<j_{2}<i_{2}<\cdots$,

(2) continuous surjections $\lambda_{\ell}: M_{i_{\ell+1}} \rightarrow M$ for $\ell \geq 0$,

(3) continuous surjections $\mu_{\ell}: M \rightarrow M_{i_{\ell}}$ for $\ell \geq 0$,

such that the following diagram $\widehat{\epsilon}$-commutes:

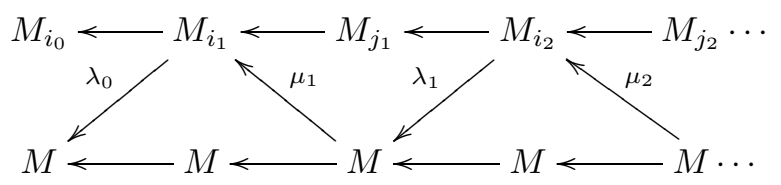

Moreover, for $\widehat{\lambda}=\left\{\lambda_{\ell}: M_{i_{\ell+1}} \rightarrow M \mid \ell \geq 0\right\}$, the induced map $S(\widehat{\lambda}): \mathcal{S}_{\mathcal{P}} \rightarrow \mathcal{S}_{\mathcal{Q}}$ is $\epsilon$-close to $\Phi$.

For $\widehat{\mu}=\left\{\mu_{\ell}: M \rightarrow M_{i_{\ell}} \mid \ell \geq 0\right\}$, the induced map $S(\widehat{\mu}): \mathcal{S}_{\mathcal{Q}} \rightarrow \mathcal{S}_{\mathcal{P}}$ is $\epsilon$-homotopic to $\Phi^{-1}$, and in particular the maps $\Phi^{-1}$ and $S(\widehat{\mu})$ are $\epsilon$-close.

REMARK 3.6. In Section [5] we compare in Theorem [5.1 the pro-groups obtained from the fundamental groups for the spaces in the top and bottom rows of (24) in Theorem 3.2 and the fact that we are given that the squares formed by the maps in $\widehat{\lambda}$ and $\widehat{\mu}$ are $\widehat{\epsilon}$-homotopic, and that the maps in $\widehat{\lambda}$ and $\widehat{\mu}$ are coverings, suffices to imply that these pro-groups are isomorphic.

Then in Section 6, we compare in Theorem 6.1 the pro-groups obtained from the fundamental groups for the spaces in the top and bottom rows of (27) in Theorem 3.5. However, in this case, it is no longer given that the maps in $\hat{\lambda}$ and $\widehat{\mu}$ are coverings. We note that the proof of Theorem 3.5 by Mardešić and Segal shows that there are simplicial spaces $N_{\ell}$, with each $N_{\ell}$ homeomorphic to $M$, such that the maps $q_{\ell+1}: M \rightarrow M$ for $\ell \geq 0$ are induced by simplicial maps $q_{\ell+1}^{\prime}: N_{\ell+1} \rightarrow N_{\ell}$. Thus, we can use the results of James W. Rogers in [71] to conclude that the squares formed by the maps in $\widehat{\lambda}$ and $\widehat{\mu}$ are in fact $\widehat{\epsilon}$-homotopic. However, it is not given that the maps in $\widehat{\lambda}$ and $\widehat{\mu}$ are locally onto, as they need not be covering maps, so that one does not have the injectivity property on fundamental groups that covering maps enjoy. Thus, an additional argument is needed to compare the pro-groups obtained from the fundamental groups for the spaces in the top and bottom rows of (27). This additional fact is given by Proposition 6.5 which shows the local onto property for maps restricted to leaves, which is then used to establish an $\epsilon$-homotopy lifting property that is sufficient to compare these pro-groups by the path-lifting property.

\section{PRO-GRoups AND GROUP CHAins}

In this section, we recall the general definition of a pro-group, and equivalence between pro-groups, following the development in [57, Chapter II, Section 2]. We then compare this notion with the group chains introduced in the previous section.

DEFINITION 4.1. A pro-group is a collection

$$
\mathcal{G}\left(G_{\lambda}, p_{\lambda^{\prime}}^{\lambda}, \Lambda\right)=\left\{p_{\lambda^{\prime}}^{\lambda}: G_{\lambda^{\prime}} \rightarrow G_{\lambda} \mid \lambda \leq \lambda^{\prime}, \lambda, \lambda^{\prime} \in \Lambda\right\}
$$

where the $G_{\lambda}$ are groups, $p_{\lambda^{\prime}}^{\lambda}$ are group homomorphisms, and $\Lambda$ is a directed index set.

A morphism $\left(f_{\gamma}, \phi\right)$ between pro-groups $\mathcal{G}\left(G_{\lambda}, p_{\lambda^{\prime}}^{\lambda}, \Lambda\right)$ and $\mathcal{G}\left(H_{\lambda}, q_{\gamma^{\prime}}^{\gamma}, \Gamma\right)$ is given by an order-preserving $\operatorname{map} \phi: \Gamma \rightarrow \Lambda$ and homomorphisms $f_{\gamma}: G_{\phi(\gamma)} \rightarrow H_{\gamma}$ such that, whenever $\gamma<\gamma^{\prime}$, then there is $\lambda \in \Lambda$, 
$\lambda \geq \phi(\gamma)$ and $\lambda \geq \phi\left(\gamma^{\prime}\right)$, for which $f_{\gamma} \circ p_{\lambda}^{\phi(\gamma)}=q_{\gamma^{\prime}}^{\gamma} \circ f_{\gamma^{\prime}} \circ p_{\lambda}^{\phi\left(\gamma^{\prime}\right)}$. That is, the following commutes:

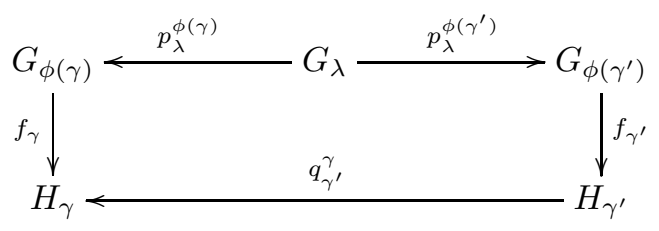

The diagram (28) is called an equalizer between the maps $f_{\gamma}$ and $f_{\gamma^{\prime}}$.

The composition of morphisms of pro-groups is again a morphism, and the operation is associative. The details of the proofs of these statements, along with the notion of equivalence between morphisms, can be found in [57, Chapter I, Section 1.1]. Also, the notion of monomorphism, epimorphism and isomorphism between pro-groups is defined there. We recall a reformulation of these notions using the results in [57, Chapter I, Sections 1.1, 1.2].

Given a morphism $\left(f_{\gamma}, \phi\right)$, a pair $(\lambda, \gamma) \in \Lambda \times \Gamma$ is said to be admissible if $\lambda \geq \phi(\gamma)$. For an admissible pair $(\lambda, \gamma)$ define the homomorphism

$$
f_{\lambda}^{\gamma}=f_{\gamma} \circ p_{\lambda}^{\phi(\gamma)}
$$

DEFINITION 4.2. [57, Chapter II, Section 2.1, Theorem 2] A morphism $\left(f_{\gamma}, \phi\right)$ between progroups $\mathcal{G}\left(G_{\lambda}, p_{\lambda^{\prime}}^{\lambda}, \Lambda\right)$ and $\mathcal{G}\left(H_{\lambda}, q_{\gamma^{\prime}}^{\gamma}, \Gamma\right)$ is a monomorphism if for every admissible pair $(\lambda, \gamma)$ for $\left(f_{\gamma}, \phi\right)$, there is an admissible pair $\left(\lambda^{\prime}, \gamma^{\prime}\right)$ for $\left(f_{\gamma}, \phi\right)$ with $\lambda^{\prime} \geq \lambda$ and $\gamma^{\prime} \geq \gamma$, such that

$$
\operatorname{Ker}\left\{f_{\lambda^{\prime}}^{\gamma^{\prime}}: G_{\lambda^{\prime}} \rightarrow H_{\gamma^{\prime}}\right\} \subset \operatorname{Ker}\left\{p_{\lambda^{\prime}}^{\lambda}: G_{\lambda^{\prime}} \rightarrow G_{\lambda}\right\}
$$

DEFINITION 4.3. [57, Chapter II, Section 2.1, Theorem 4] A morphism $\left(f_{\gamma}, \phi\right)$ between progroups $\mathcal{G}\left(G_{\lambda}, p_{\lambda^{\prime}}^{\lambda}, \Lambda\right)$ and $\mathcal{G}\left(H_{\lambda}, q_{\gamma^{\prime}}^{\gamma}, \Gamma\right)$ is an epimorphism if for every admissible pair $(\lambda, \gamma)$ for $\left(f_{\gamma}, \phi\right)$, there is an admissible pair $\left(\lambda^{\prime}, \gamma^{\prime}\right)$ for $\left(f_{\gamma}, \phi\right)$ with $\lambda^{\prime} \geq \lambda$ and $\gamma^{\prime} \geq \gamma$, such that

$$
\text { Image }\left\{q_{\gamma^{\prime}}^{\gamma}: H_{\gamma^{\prime}} \rightarrow H_{\gamma}\right\} \subset \text { Image }\left\{f_{\lambda}^{\gamma}: G_{\lambda} \rightarrow H_{\gamma}\right\} \text {. }
$$

The following characterization of isomorphism of pro-groups is then equivalent to Morita's Lemma:

DEFINITION 4.4. [57, Chapter II, Section 2.2, Theorem 6] A morphism $\left(f_{\gamma}, \phi\right)$ between progroups $\mathcal{G}\left(G_{\lambda}, p_{\lambda, \lambda^{\prime}}, \Lambda\right)$ and $\mathcal{G}\left(H_{\lambda}, q_{\gamma, \gamma^{\prime}}, \Gamma\right)$ is an isomorphism if and only if $\left(f_{\gamma}, \phi\right)$ is a monomorphism and an epimorphism.

For our purposes, the ordered sets $\Lambda$ and $\Gamma$ above will always be assumed to be a subset of the non-negative integers $\mathbb{N}$ with the natural order.

Finally, we compare the notions of group chains and the pro-groups they determine. Let $\mathcal{G}=\left\{G_{\ell}\right\}$ and $\mathcal{H}=\left\{H_{\ell}\right\}$ be two group chains, which determine pro-groups

$$
\begin{aligned}
& \mathcal{G}\left(G_{\ell}, p_{\ell^{\prime}}^{\ell}\right)=\left\{p_{\ell^{\prime}}^{\ell}: G_{\ell^{\prime}} \rightarrow G_{\ell} \mid \ell^{\prime} \geq \ell \geq 0, \ell, \ell^{\prime} \in \mathbb{N}\right\} \\
& \mathcal{G}\left(H_{\ell}, q_{\ell^{\prime}}^{\ell}\right)=\left\{q_{\ell^{\prime}}^{\ell}: H_{\ell^{\prime}} \rightarrow H_{\ell} \mid \ell^{\prime} \geq \ell \geq 0, \ell, \ell^{\prime} \in \mathbb{N}\right\} .
\end{aligned}
$$

where the maps $p_{\ell^{\prime}}^{\ell}$ and $q_{\ell^{\prime}}^{\ell}$ are given by the inclusions of subgroups.

Suppose the group chains $\mathcal{G}$ and $\mathcal{H}$ are equivalent in the sense of Definition 2.1. Then there are infinite increasing sequences $\left\{\ell_{k}\right\}$ and $\left\{j_{k}\right\}$, and inclusion maps

$$
\lambda_{k}: K_{2 k+2}=G_{\ell_{k+1}} \subset K_{2 k+1}=H_{j_{k}} \quad \text { and } \quad \mu_{k}: K_{2 k+1}=H_{j_{k}} \subset K_{2 k}=G_{\ell_{k}} .
$$

Introduce the group chains $\mathcal{A}=\left\{A_{k} \equiv G_{\ell_{k}} \mid k \geq 1\right\}$ and $\mathcal{B}=\left\{B_{k} \equiv H_{j_{k}} \mid k \geq 1\right\}$. Then we obtain a pro-group $\mathcal{G}\left(A_{k}, \iota_{k^{\prime}}^{k}\right)$, where $\iota_{k^{\prime}}^{k}: A_{k^{\prime}} \subset A_{k}$ is the inclusion map. It is immediate that $\mathcal{G}\left(G_{\ell}, p_{\ell^{\prime}}^{\ell}\right)$ and $\mathcal{G}\left(A_{k}, \iota_{k^{\prime}}^{k}\right)$ are isomorphic pro-groups. Likewise, form the pro-group $\mathcal{G}\left(B_{k}, \xi_{k^{\prime}}^{k}\right)$ where $\xi_{k^{\prime}}^{k}: B_{k^{\prime}} \subset B_{k}$ is the inclusion map. It is immediate that $\mathcal{G}\left(H_{\ell}, q_{\ell^{\prime}}^{\ell}\right)$ and $\mathcal{G}\left(B_{k}, \xi_{k^{\prime}}^{k}\right)$ are isomorphic pro-groups. 
For $k \geq 1$, set $\phi(k)=k+1$, and let $f_{k}=\lambda_{k}: A_{k+1} \rightarrow B_{k}$. Then the map of pro-groups $\left(f_{k}, \phi\right)$ is a monomorphism, as each $\lambda_{k}$ is a monomorphism. This map is also an epimorphism, which follows using the maps $\left\{\mu_{k}\right\}$. It follows that the pro-groups $\mathcal{G}\left(G_{\ell}, p_{\ell^{\prime}}^{\ell}\right)$ and $\mathcal{G}\left(H_{\ell}, q_{\ell^{\prime}}^{\ell}\right)$ are isomorphic.

If the group chains $\mathcal{G}$ and $\mathcal{H}$ are equivalent in the sense of Definition 2.2, then there exists a sequence $\left(g_{\ell}\right)_{\ell>0}$ where each $g_{\ell} \in G_{0}$ and $g_{\ell} G_{\ell}=g_{\ell+1} G_{\ell}$ for all $\ell \geq 0$, so that the groups chains $\mathcal{G}^{\left(g_{\ell}\right)}=\left\{g_{\ell} G_{\ell} g_{\ell}^{-1}\right\}_{\ell \geq 0}$ and $\mathcal{H}$ are equivalent. Define

$$
\lambda_{k}(g)=g_{\ell_{k+1}} g g_{\ell_{k+1}}^{-1} \text { for } g \in G_{\ell_{k+1}} \quad, \quad \mu_{k}(h)=g_{j_{k}}^{-1} h g_{j_{k}} \text { for } h \in H_{j_{k}},
$$

then as before, this yields an isomorphism between the pro-groups $\mathcal{G}\left(G_{\ell}, p_{\ell^{\prime}}^{\ell}\right)$ and $\mathcal{G}\left(H_{\ell}, q_{\ell^{\prime}}^{\ell}\right)$.

Next, suppose that we have an isomorphism $\left(f_{\gamma}, \phi\right)$ between pro-groups $\mathcal{G}\left(G_{\ell}, p_{\ell^{\prime}}^{\ell}\right)$ and $\mathcal{G}\left(H_{\ell}, q_{\ell^{\prime}}^{\ell}\right)$. Then we have monomorphisms $f_{\ell}: G_{\phi(\ell)} \rightarrow H_{\ell}$. Define $H_{\ell}^{\prime}=f_{\ell}\left(G_{\phi(\ell)}\right)$, then by the commutative properties in diagram (28) and the isomorphism conditions (30) and (31), we obtain a group chain

$$
H_{0} \supset H_{1} \supset H_{1}^{\prime} \supset H_{2} \supset H_{2}^{\prime} \supset \cdots \text {. }
$$

It follows that the group chains $\mathcal{H}=\left\{H_{\ell}\right\}$ and $\mathcal{H}^{\prime}=\left\{H_{\ell}^{\prime}\right\}$ are equivalent in the sense of Definition 2.1

Now suppose that the pro-groups are obtained from group chains $\mathcal{G}$ and $\mathcal{H}$ as above. For simplicity, assume that $G_{0}=H_{0}$. Assume that the pro-groups are isomorphic, then by the above, we have that $\mathcal{H}$ and $\mathcal{H}^{\prime}$ are equivalent group chains. However, this does not imply that the group chains $\mathcal{G}$ and $\mathcal{H}$ in $G_{0}$ are conjugate equivalent, as the conditions in Definition 2.2 require that each monomorphism $f_{\ell}$ be implemented by conjugation by some element $g_{\ell} \in G_{0}$. In other words, given two isomorphic subgroups of a group, they need not be in the same conjugacy class. If we consider the weaker notion of return equivalence in Definition 2.6. then the requirement becomes that there exists some $\ell_{0}>0$ such that the maps $f_{\ell}$ are implemented by a conjugacy for $\ell \geq \ell_{0}$ and this need not hold in general either. Thus, the notion of isomorphism between the pro-groups defined by group chains $\mathcal{G}$ and $\mathcal{H}$ may be weaker than the notion of return equivalence between the group chains.

QUESTION 4.5. Is there a natural algebraic condition on a group $G$, which implies that two group chains $\mathcal{G}$ and $\mathcal{H}$ in $G$ which are isomorphic as pro-groups must be return equivalent?

\section{PRO-FUndAMENTAL GROUPS OF WEAK SOLENOIDS}

A presentation for a continuum $\mathfrak{M}$ can be viewed as a particular type of shape expansion for $\mathfrak{M}$, and so defines its pro-homotopy type, in the sense of Mardešić and Segal [57, or in the foundational work by Bousfield and Kan [12. One can apply various functors to this shape expansion to obtain invariants of $\mathfrak{M}$. Mardešić and Segal show in [57, Chapter II, Section 3.1] that the pro-homology groups pro- $H_{k}(\mathfrak{M})$ for $k>0$ are well-defined, and show in [57, Chapter II, Section 3.3] that the pro-homotopy groups pro- $\pi_{k}(\mathfrak{M}, z)$ for $k>1$ are well-defined.

The definition of the pro-fundamental group pro- $\pi_{1}(\mathfrak{M}, z)$ for a weak solenoid requires extra care, due to basepoint issues. If one assumes that there is a shape expansion which preserves basepoints, and that maps between expansions also preserves basepoints, then the results of [57, Chapter II, Section 3.3 show that the pro-fundamental group is well-defined. However, the maps between presentations in Section 3 above are not assumed to preserve basepoints. The work of Fox in [40] introduces the notion of tropes to circumvent these difficulties, and define covering spaces for shape approximations. This topic is addressed further in the work of Mardešić and Matijević [58], and the work of Eda, Mandić and Matijević in 34] illustrates some of the subtleties of the issues involved. In any case, this approach does not suffice for the study of the equivalence of pro-fundamental groups for the inverse limits spaces we consider in this work. Instead, we develop an approach based on the fact that the shape expansions we work with are derived from a matchbox manifold, so that we can use the geometry of the leaves of the foliation of this space to show the pro-groups are well-defined.

Now assume that there is a solenoidal presentation $\mathcal{P}$ and a homeomorphism $\Phi_{\mathcal{P}}: \mathfrak{M} \rightarrow \mathcal{S}_{\mathcal{P}}$. Choose a basepoint $z \in \mathfrak{M}$, then set $x=\Phi_{\mathcal{P}}(z)$. Let $\Pi_{\ell}^{\mathcal{P}}: \mathcal{S}_{\mathcal{P}} \rightarrow M_{\ell}$ be the projection map onto the factor 
space $M_{\ell}$, set $x_{\ell}=\Pi_{\ell}^{\mathcal{P}}(x) \in M_{\ell}$ and let $X(\mathcal{P})_{\ell}=\left(\Pi_{\ell}^{\mathcal{P}}\right)^{-1}\left(x_{\ell}\right) \subset \mathcal{S}_{\mathcal{P}}$. Define the pro-group

$$
\mathcal{G}(\mathcal{P}, x)=\left\{p_{\ell+1}: \pi_{1}\left(M_{\ell+1}, x_{\ell+1}\right) \rightarrow \pi_{1}\left(M_{\ell}, x_{\ell}\right) \mid \ell \geq 0\right\}
$$

where by a small abuse of notation, we let $p_{\ell+1}$ also denote the induced map on fundamental groups.

We then have the following result, which can be considered a formal restatement of results in [38]:

THEOREM 5.1. Let $\mathfrak{M}$ be an $M$-like matchbox manifold, and let $\mathcal{P}$ be a presentation for which there is a homeomorphism $\Phi_{\mathcal{P}}: \mathfrak{M} \rightarrow \mathcal{S}_{\mathcal{P}}$. Choose $z \in \mathfrak{M}$ and set $x=\Phi_{\mathcal{P}}(z)$. Then the isomorphism class of the pro-group pro- $\pi_{1}(\mathfrak{M}, z)$ derived from $\mathcal{G}(\mathcal{P}, x)$ is independent of the choice of presentation $\mathcal{P}$ and basepoint $x$, and thus is invariant of the homeomorphism class of $\mathfrak{M}$.

Proof. Suppose that $\mathcal{Q}$ is another presentation for $\mathfrak{M}$ as a weak solenoid, then we are given the hypotheses and conclusions of Theorem 3.2. We use the notation there. Choose a basepoint $z \in \mathfrak{M}$, let $x=\Phi_{\mathcal{P}}(z)$ and $y=\Phi_{\mathcal{Q}}(z)$. Set $\Phi=\Phi_{\mathcal{Q}}^{-1} \circ \Phi_{\mathcal{P}}$.

For the pro-morphisms $\widehat{\lambda}$ and $\widehat{\mu}$ obtained from the maps in diagram (24) which $\widehat{\epsilon}$-commutes, the induced map $S(\widehat{\lambda})$ is $\epsilon$-homotopic to $\Phi$, and similarly the induced map $S(\widehat{\mu})$ is $\epsilon$-homotopic to $\Phi^{-1}$.

For $\ell \geq 0$ set $x_{\ell}=\Pi_{\ell}^{\mathcal{P}}(x) \in M_{\ell}$ and $y_{\ell}=\Pi_{\ell}^{\mathcal{Q}}(y) \in N_{\ell}$, then we have:

$$
d_{N_{j_{\ell}}}\left(\lambda_{\ell}\left(x_{i_{\ell+1}}\right), y_{j_{\ell}}\right) \leq \epsilon_{\ell} \text { and } d_{M_{i_{\ell}}}\left(\mu_{\ell}\left(y_{j_{\ell}}\right), x_{i_{\ell}}\right) \leq \epsilon_{\ell} .
$$

The $\epsilon$-homotopy from $S(\widehat{\lambda})$ to $\Phi$ defines a path from the basepoint $\lambda_{\ell}\left(x_{i_{\ell+1}}\right)$ to the basepoint $y_{j_{\ell}}$, and similarly from the basepoint $\mu_{\ell}\left(y_{j_{\ell}}\right)$ to the basepoint $x_{i_{\ell}}$. Moreover, this homotopy induces a homotopy of paths at these basepoints, so we obtain from (24) a commutative diagram:

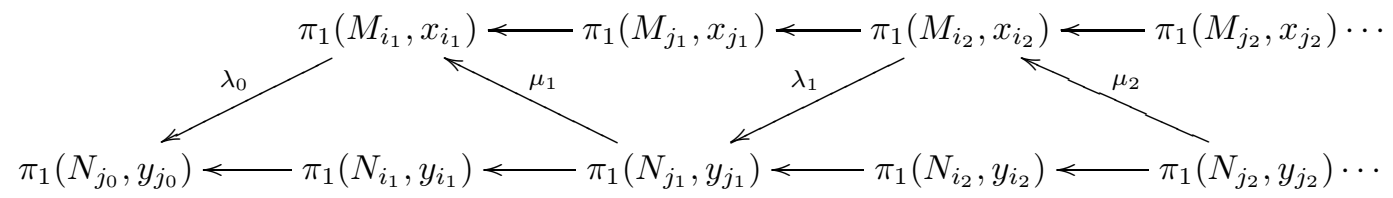

where we again abuse notation, by denoting a map and its induced map on fundamental groups the same. All maps in (36) are monomorphisms, as they are induced from covering maps.

We check that the conditions of Definitions 4.2 and 4.3 are satisfied for $G_{i}=\pi_{1}\left(M_{i}, x_{i}\right)$ and $H_{j}=$ $\pi_{1}\left(N_{j}, y_{j}\right)$. We first translate the conditions (30) and the conditions (31) into the nomenclature of diagram (36). The corresponding indexing sets are $\Lambda=\left\{i_{1}, i_{2}, \ldots\right\}$ and $\Gamma=\left\{j_{1}, j_{2}, j_{3}, \ldots\right\}$. Recall that these indices are chosen so that $j_{\ell}<i_{\ell}<j_{\ell+1}<i_{\ell+1}$ for all $\ell \geq 0$.

The maps $f_{\lambda}$ correspond to the maps $\lambda_{\ell}=G_{i_{\ell+1}} \rightarrow H_{j_{\ell}}$ so we have $\phi\left(j_{\ell}\right)=i_{\ell+1}$.

The admissible condition $\lambda \geq \phi(\gamma)$ then becomes, for $\lambda=k$ and $\gamma=\ell$, that $i_{k} \geq i_{\ell+1}$ so by our choice of the sequences, that $k \geq \ell+1$.

The maps $f_{\gamma}: G_{\phi(\gamma)} \rightarrow H_{\gamma}$ correspond to the maps $\lambda_{\ell}: G_{i_{\ell+1}} \rightarrow H_{j_{\ell}}$, and so the maps $f_{\lambda}^{\gamma}=f_{\gamma} \circ p_{\lambda}^{\phi(\gamma)}$ defined in (29) correspond to the maps $f_{k}^{\ell}=\lambda_{\ell} \circ p_{k}^{\phi\left(j_{\ell}\right)}$. Then the monomorphism condition (30) becomes

$$
\operatorname{Ker}\left\{\lambda_{\ell^{\prime}} \circ p_{k^{\prime}}^{\phi\left(j_{\ell^{\prime}}\right)}: G_{k^{\prime}} \rightarrow H_{\ell^{\prime}}\right\} \subset \operatorname{Ker}\left\{p_{k^{\prime}}^{k}: G_{k^{\prime}} \rightarrow G_{k}\right\}
$$

and the epimorphism condition (31) becomes

$$
\text { Image }\left\{q_{\ell^{\prime}}^{\ell}: H_{\ell^{\prime}} \rightarrow H_{\ell}\right\} \subset \text { Image }\left\{\lambda_{\ell} \circ p_{k}^{\phi\left(j_{\ell}\right)}: G_{k} \rightarrow H_{\ell}\right\} .
$$

The induced maps $\lambda_{\ell}$ and $p_{\ell}$ are monomorphisms, as they are induced by covering maps, so condition (37) is satisfied. To show condition (38), we use the commutativity of the diagram (36) which implies that $q_{\ell^{\prime}}^{\ell}=\lambda_{\ell} \circ p_{i_{\ell^{\prime}}}^{i_{\ell+1}} \circ \mu_{\ell^{\prime}}$ so that (38) is satisfied for $k=\ell^{\prime} \geq \ell+1$.

Thus, the pro-morphisms $\widehat{\lambda}$ and $\widehat{\mu}$ induce isomorphisms between the pro-groups defined by the top and bottom rows of (36). This shows that the pro-group $\mathcal{G}(\mathcal{P}, x)$ defined in (34) is independent of the choice of presentation $\mathcal{P}$. 
It remains to show that for two choices $z, z^{\prime} \in \mathfrak{M}$, the pro-groups $\mathcal{G}(\mathcal{P}, x)$ and $\mathcal{G}\left(\mathcal{P}, x^{\prime}\right)$ are isomorphic for $x=\Phi_{\mathcal{P}}(z)$ and $x^{\prime}=\Phi_{\mathcal{P}}\left(z^{\prime}\right)$. By Remark 3.3, we can assume that $x^{\prime} \in \mathfrak{X}_{0}$. Then by Theorem 2.7 the group chains $\mathcal{G}_{x}$ and $\mathcal{G}_{x^{\prime}}$ are conjugate return equivalent in the sense of Definition 2.5.

Then as in the discussion at the end of Section 4, observe that the conjugation maps in Definition 2.2 induce isomorphisms between the subgroups $G_{\ell}$ and $H_{\ell}$ for $\ell>0$, and the notion of equivalence of group chains in Definition 2.1 induces an isomorphism of the pro-groups $\mathcal{G}(\mathcal{P}, x)$ and $\mathcal{G}\left(\mathcal{P}, x^{\prime}\right)$ they determine, as was to be shown.

REMARK 5.2. We note a curious aspect of the conclusion of Theorem [5.1. The isomorphism class pro- $\pi_{1}(\mathfrak{M}, z)$ is independent of the choice of basepoint used to define the group chain $\mathcal{G}^{x}$, for $x=\Phi_{\mathcal{P}}(z)$, while by Remark 2.4 the kernel of the associated group chain $K\left(\mathcal{G}^{x}\right)$ may depend on the choice of $x$. Thus, there is some loss of information when we pass from a group chain $\mathcal{G}^{x}$ to the pro-group it determines. The reason is simply that the group chain $\mathcal{G}^{x}$ contains the information on how the groups are embedded in $G_{0}$, while the pro-group pro- $\pi_{1}(\mathfrak{M}, x)$ does not.

\section{Pro-fundamental groups of M-Like spaces}

In this section we prove the following, which can be considered the main result of this work.

THEOREM 6.1. Let $\mathfrak{M}$ be a matchbox manifold which is $M$-like. Then pro- $\pi_{1}(\mathfrak{M}, z)$ is proisomorphic to the pro-group defined by maps $\left\{g_{\ell}: G \rightarrow G \mid \ell \geq 0\right\}$, where $G=\pi_{1}(M, y)$.

Proof. The idea is to use Theorem 3.4 to obtain a diagram analogous to (36), and then follow the outline of the proof of Theorem 5.1. This requires that we first choose basepoints that "almost commute", so the pro-morphisms $\widehat{\lambda}$ and $\widehat{\mu}$ are well-defined. The main issue then is to show the maps satisfy the conditions (37) and (38). It follows from Theorem 3.5 that the maps in $\widehat{\mu}$ induce commutative squares in the diagram (27). Unfortunately, the maps $\hat{\lambda}$ in the diagram (27) are only known to $\hat{\epsilon}$-commute, and neither collection of maps $\widehat{\lambda}$ and $\widehat{\mu}$ are known to be coverings. Thus, another approach is required, and we use a technique that is analogous to the approach of Rogers and Tollefson in [70, Section 3].

We first recall some basic results about the leafwise geometry of foliated spaces and matchbox manifolds, as discussed in detail for example in the works [13, 16, 19.

THEOREM 6.2. Let $\mathfrak{M}$ be a smooth matchbox manifold with foliation $\mathcal{F}_{\mathfrak{M}}$. Then there exists a leafwise Riemannian metric for $\mathcal{F}_{\mathfrak{M}}$, such that for each $x \in \mathfrak{M}$, the leaf $L_{x}$ inherits the structure of a complete Riemannian manifold with bounded geometry, and the Riemannian metric and its covariant derivatives depend continuously on $x$.

Bounded geometry implies, for example, that for each $x \in \mathfrak{M}$, there is a leafwise exponential $\operatorname{map} \exp _{x}^{\mathcal{F}}: T_{x} \mathcal{F}_{\mathfrak{M}} \rightarrow L_{x}$ which is a surjection, and the composition with the inclusion map, $\exp _{x}^{\mathcal{F}}: T_{x} \mathcal{F}_{\mathfrak{M}} \rightarrow L_{x} \subset \mathfrak{M}$, depends continuously on $x$ in the compact-open topology on maps.

Each leaf $L \subset \mathfrak{M}$ has a complete path-length metric, induced from the leafwise Riemannian metric:

$$
d_{\mathcal{F}}(x, y)=\inf \left\{\|\gamma\| \mid \gamma:[0,1] \rightarrow L \text { is piecewise } \mathrm{C}^{1}, \gamma(0)=x, \gamma(1)=y, \gamma(t) \in L \quad \forall 0 \leq t \leq 1\right\}
$$

where $\|\gamma\|$ denotes the path-length of the piecewise $C^{1}$-curve $\gamma(t)$. If $x, y \in \mathfrak{M}$ are not on the same leaf, then set $d_{\mathcal{F}}(x, y)=\infty$. For each $x \in \mathfrak{M}$ and $r>0$, let $D_{\mathcal{F}}(x, r)=\left\{y \in L_{x} \mid d_{\mathcal{F}}(x, y) \leq r\right\}$.

For each $x \in \mathfrak{M}$, the Gauss Lemma implies that there exists $\lambda_{x}>0$ such that $D_{\mathcal{F}}\left(x, \lambda_{x}\right)$ is a strongly convex subset for the metric $d_{\mathcal{F}}$. That is, for any pair of points $y, y^{\prime} \in D_{\mathcal{F}}\left(x, \lambda_{x}\right)$ there is a unique shortest geodesic segment in $L_{x}$ joining $y$ and $y^{\prime}$ and contained in $D_{\mathcal{F}}\left(x, \lambda_{x}\right)$. This standard concept of Riemannian geometry is discussed in detail in [11, and in [27, Chapter 3, Proposition 4.2]. Then for all $0<\lambda<\lambda_{x}$ the disk $D_{\mathcal{F}}(x, \lambda)$ is also strongly convex. Then we have:

LEMMA 6.3. There exists $\lambda_{\mathcal{F}}>0$ such that for all $x \in \mathfrak{M}, D_{\mathcal{F}}\left(x, \lambda_{\mathcal{F}}\right)$ is strongly convex. 
Choose a presentation $\mathcal{Q}=\left\{q_{\ell+1}: M \rightarrow M \mid \ell \geq 0\right\}$ as in Theorem 3.4. The manifold $M$ is assumed to be smooth and without boundary, and choose a Riemannian metric $d_{M}$ for $M$. Let $\lambda_{M}>0$ be such that for $u \in M$, the geodesic ball $B_{M}\left(u, \lambda_{M}\right) \subset M$ is strongly convex.

Give $\mathcal{S}_{\mathcal{Q}}$ the metric (19), where each factor space $Y_{\ell}=M$ is given the metric $d_{M}$.

Choose a presentation $\mathcal{P}=\left\{p_{\ell+1}: M_{\ell+1} \rightarrow M_{\ell} \mid \ell \geq 0\right\}$ for which there is a homeomorphism $\Phi_{\mathcal{P}}: \mathfrak{M} \cong \mathcal{S}_{\mathcal{P}}$, where each $M_{\ell}$ is a compact connected $n$-dimensional manifold without boundary, and each $p_{\ell+1}$ is a covering map of finite degree greater than one. Choose a Riemannian metric on $M_{0}$ such that every geodesic ball of radius at most $\epsilon^{\prime \prime}>0$ is strongly convex, then for $\ell>0$, let $M_{\ell}$ have the Riemannian metric induced by the covering map $p_{\ell}^{0}: M_{\ell} \rightarrow M_{0}$ in (7). Give $\mathcal{S}_{\mathcal{P}}$ the metric as in (19). It then follows that for each $b \in M_{0}$ and closed ball $B_{M_{0}}\left(b, \epsilon^{\prime \prime} / 2\right) \subset M_{0}$, the inverse image $\Pi_{0}^{-1}\left(B_{M_{0}}\left(b, \epsilon^{\prime \prime} / 2\right)\right) \subset \mathcal{S}_{\mathcal{P}}$ is a chart for the foliation $\mathcal{F}_{\mathcal{P}}$ on the inverse limit $\mathcal{S}_{\mathcal{P}}$. The proof by McCord in [59] that weak solenoids are foliated spaces used just such charts. Also, note that each leaf $L \subset \mathcal{S}_{\mathcal{P}}$ is a covering of $M_{0}$. The lift of the Riemannian metric on $M_{0}$ to $L$ defines a path length metric on each leaf $L$ of $\mathcal{F}_{\mathcal{P}}$.

Moreover, the homeomorphism $\Phi_{\mathcal{P}}$ preserves the path components of $\mathfrak{M}$ and $\mathcal{S}_{\mathcal{P}}$ so for each leaf $L \subset \mathfrak{M}$ the restriction $\Phi_{\mathcal{P}} \mid L$ is a homeomorphism onto a leaf of $\mathcal{F}_{\mathcal{P}}$. Moreover, the compactness of $\mathfrak{M}$ implies that each such restriction is uniformly continuous for the leafwise metrics on $\mathcal{F}_{\mathfrak{M}}$ and $\mathcal{F}_{\mathcal{P}}$. Thus, we can choose $\epsilon^{\prime \prime}>0$ sufficiently small so that for each $w \in \mathcal{S}_{\mathcal{P}}$ the leafwise ball $B_{\mathcal{S}_{\mathcal{P}}}\left(w, \epsilon^{\prime \prime}\right) \subset L_{z}$ is contained in the image $\Phi\left(B_{\mathfrak{M}}\left(u, \lambda_{\mathcal{F}}\right)\right)$, where $u=\Phi_{\mathcal{P}}^{-1}(w)$.

By Theorem 3.5, for $\epsilon=\min \left\{\epsilon^{\prime} / 4, \epsilon^{\prime \prime} / 4\right\}$, there exists a sequence $\widehat{\epsilon}=\left\{\epsilon \geq \epsilon_{0}>\epsilon_{1}>\cdots\right\}$ descending to zero, and

(1) an intertwined increasing sequence $0 \leq j_{0}<i_{0}<j_{1}<i_{1}<j_{2}<i_{2}<\cdots$,

(2) continuous surjections $\widehat{\lambda} \equiv\left\{\lambda_{\ell}: M_{i_{\ell+1}} \rightarrow M \mid \ell \geq 0\right\}$,

(3) continuous surjections $\widehat{\mu} \equiv\left\{\mu_{\ell}: M \rightarrow M_{i_{\ell}} \mid \ell \geq 0\right\}$,

(4) a homeomorphism $\Phi_{\mathcal{Q}}: \mathfrak{M} \rightarrow \mathcal{S}_{\mathcal{Q}}$,

so that the diagram (39) below $\widehat{\epsilon}$-commutes, where for the convenience of the arguments to follow we use $N_{\ell}=M$ so that the indexing is indicated:

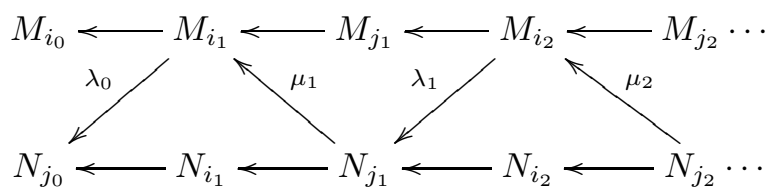

This means that for each $\ell>0$, we have

$$
\begin{aligned}
& d_{M}\left(\lambda_{\ell} \circ \mu_{\ell+1}(y), q_{j_{\ell+1}}^{j_{\ell}}(y)\right) \leq \epsilon_{\ell} \text { for all } y \in N_{j_{\ell+1}}=M \\
& d_{M_{i_{\ell}}}\left(\mu_{\ell} \circ \lambda_{\ell}(x), p_{i_{\ell+1}}^{i_{\ell}}(x)\right) \leq \epsilon_{\ell} \text { for all } y \in M_{i_{\ell+1}} .
\end{aligned}
$$

Choose $z \in \mathfrak{M}$, let $x=\Phi_{\mathcal{P}}(z)$ and $y=\Phi_{\mathcal{Q}}(z)$. Set $x_{\ell}=\Pi_{\ell}^{\mathcal{P}}(x) \in M_{\ell}$ and $y_{\ell}=\Pi_{\ell}^{\mathcal{Q}}(y) \in M$.

By (40) we have that $\mu_{\ell}\left(y_{j_{\ell}}\right) \in B_{M_{i_{\ell}}}\left(x_{i_{\ell}}, \epsilon_{i_{\ell}}\right)$ which is a contractible disk. Thus, we obtain a welldefined map $\mu_{\ell}: \pi_{1}\left(M, y_{j_{\ell}}\right) \rightarrow \pi_{1}\left(M_{i_{\ell}}, x_{i_{\ell}}\right)$ where we again use the same notation for the map on spaces and the induced map on homotopy groups. Similarly, (41) implies that there well-defined map $\lambda_{\ell}: \pi_{1}\left(M_{i_{\ell+1}}, x_{i_{\ell+1}}\right) \rightarrow \pi_{1}\left(M, y_{i_{\ell}}\right)$. Thus we obtain the following diagram:

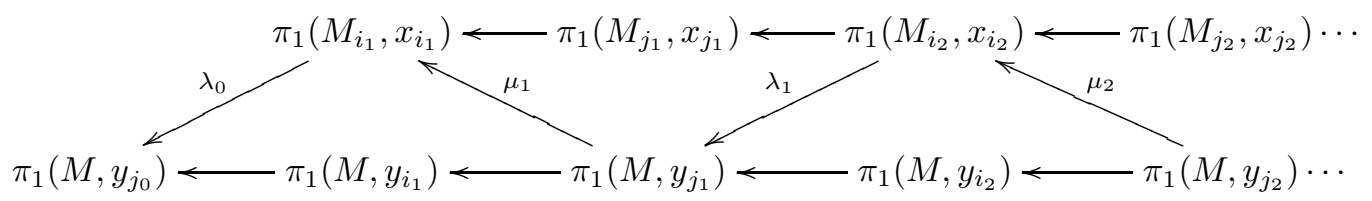


The maps in the top row of (42) are all monomorphisms as they are induced by covering maps, and the squares formed by the maps in $\widehat{\mu}$ are commutative as the induced map $S(\widehat{\mu}): \mathcal{S}_{\mathcal{Q}} \rightarrow \mathcal{S}_{\mathcal{P}}$ is $\epsilon$-homotopic to $\Phi^{-1}$. We claim that the squares formed by the maps in $\hat{\lambda}$ on fundamental groups in (42) are commutative, and then show that the properties (37) and (38) are satisfied.

The idea of the proof of these claims is as follows. Let $L \subset \mathfrak{M}$ be a leaf of $\mathcal{F}_{\mathfrak{M}}$, endowed with the topology defined by the path-length metric (which is called the model $\widehat{L}$ for $L$ in [70, Section 3]). Recall that $\Phi_{\mathcal{P}}(L)$ is a leaf of $\mathcal{F}_{\mathcal{P}}$ as the homeomorphism $\Phi_{\mathcal{P}}$ preserves path components, and then the restriction $\Pi_{\ell}^{\mathcal{P}} \mid \Phi_{\mathcal{P}}(L)$ is a covering map by construction, for each $\ell>0$. Thus, the restriction $\Pi_{\ell}^{\mathcal{P}} \circ \Phi_{\mathcal{P}}: L \rightarrow M_{\ell}$ is a covering map. However, the composition $\Pi_{\ell}^{\mathcal{Q}} \circ \Phi_{\mathcal{Q}}: L \rightarrow M$ need not be a covering map. What we claim is that this restriction is an approximate covering map, in that it satisfies an approximate lifting property, to be defined precisely below.

There have been many works studying the properties of $\epsilon$-maps between compact manifolds, starting with those of Eilenberg [36] and Ganea [42, and more recently by Chapman and Ferry [14, and Ferry [37. The idea in the following, is to adapt some of the more elementary ideas from these works to the restrictions $\Pi_{\ell}^{\mathcal{Q}} \circ \Phi_{\mathcal{Q}} \mid L$. There is an immediate difficulty, in that an $\epsilon$-map $f_{\epsilon}: \mathfrak{M} \rightarrow M$ is assumed to be onto, but it is not given that the restriction $\Pi_{\ell}^{\mathcal{Q}} \circ \Phi_{\mathcal{Q}} \mid L$ is locally onto. However, this property is proved in Proposition 6.4 below. Recall that $\lambda_{\mathcal{F}}>0$ is chosen so that for all $x \in \mathfrak{M}$, the leafwise closed disk $D_{\mathcal{F}}\left(x, \lambda_{\mathcal{F}}\right)$ is strongly convex. Also, recall that $\lambda_{M}>0$ was chosen so that for every $u \in M$, the closed disk $D_{M}\left(u, \lambda_{M}\right) \subset M$ is strictly convex.

PROPOSITION 6.4. [19, Proposition A.1] Let $\mathfrak{M}$ be a matchbox manifold with leafwise Riemannian metric on $\mathcal{F}_{\mathfrak{M}}$ and let $M$ be a closed Riemannian manifold. Then there exists $0<\epsilon_{\mathcal{F}} \leq \lambda_{\mathcal{F}} / 4$

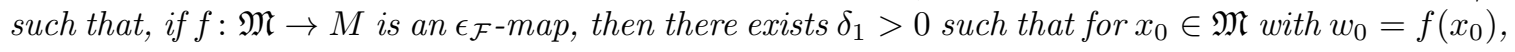

$$
D_{M}\left(w_{0}, \delta_{1}\right) \subset f\left(D_{\mathcal{F}}\left(x_{0}, \lambda_{\mathcal{F}} / 2\right)\right) .
$$

Thus, (43) implies that every sufficiently small disk in $M$ is contained in the image of a strictly convex disk in some leaf of $\mathcal{F}_{\mathfrak{M}}$.

PROPOSITION 6.5. There exists $\ell_{2}>0$ such that for $\ell \geq \ell_{2}$ the map $\Pi_{\ell}^{Q} \circ \Phi_{\mathcal{Q}}: \mathfrak{M} \rightarrow M$ satisfies an approximate homotopy lifting property. That is, given an integer $k \geq 1$, constant $0<\delta_{2}<\lambda_{M}$ and $x \in \mathfrak{M}$, let $L$ be the leaf through $x$, and given a continuous map $\sigma:[0,1]^{k} \rightarrow M$ with $\sigma(\overrightarrow{0})=$ $y_{\ell}=\Pi_{\ell}^{Q} \circ \Phi_{\mathcal{Q}}(x)$, then there exists a continuous map $\widehat{\sigma}:[0,1]^{k} \rightarrow L$ with $\widehat{\sigma}(\overrightarrow{0})=x$, for which

$$
d_{M}\left(\Pi_{\ell}^{Q} \circ \Phi_{\mathcal{Q}} \circ \widehat{\sigma}(\vec{v}), \sigma(\vec{v})\right) \leq \delta_{2} \quad \text { for all } \vec{v} \in[0,1]^{k} .
$$

Moreover, the maps $\sigma$ and $\Pi_{\ell}^{Q} \circ \Phi_{\mathcal{Q}} \circ \widehat{\sigma}$ are $\delta_{2}$-homotopic.

Proof. Choose $0<\epsilon_{2}<\lambda_{\mathcal{F}} / 4$, then there exists $\ell_{2}>0$ sufficiently large so that $\ell \geq \ell_{2}$ implies that the composition $\Pi_{\ell}^{Q} \circ \Phi_{\mathcal{Q}}$ is an $\epsilon_{2}$-map. Fix a choice of $\ell \geq \ell_{2}$.

Let $\delta_{2} \leq \lambda_{M}$ be as in Proposition 6.4 so that for all $x_{0} \in \mathfrak{M}$ with $w_{0}=f\left(x_{0}\right)$, then

$$
D_{M}\left(w_{0}, \delta_{2}\right) \subset \Pi_{\ell}^{Q} \circ \Phi_{\mathcal{Q}}\left(D_{\mathcal{F}}\left(x_{0}, \epsilon_{2}\right)\right) .
$$

Let $B\left(\vec{v}, \delta_{3}\right)$ denote the ball of radius $\delta_{3}$ in $\mathbb{R}^{k}$ for the Euclidean metric on $\mathbb{R}^{k}$. Then choose $\delta_{3}>0$ such that for any $\vec{v} \in[0,1]^{k}$, we have $\sigma\left(B\left(\vec{v}, \delta_{3}\right) \cap[0,1]^{k}\right) \subset B_{M}\left(\sigma(\vec{v}), \delta_{2}\right)$.

Choose $n_{3}>0$ so that the "small cube"' $\left[0,1 / n_{3}\right]^{k}$ has diameter less than $\delta_{3}$.

Starting with the given value $\sigma(0)=y_{\ell} \in M$ we use the above choices to recursively construct the lifting $\widehat{\sigma}$, small cube by small cube for a cubical decomposition of $[0,1]^{k}$. This is a standard construction, usually proved for the assumption that the target space $M$ is locally contractible, with the only nuance being the estimates on the control of the maps. We omit the details.

We now return to the proof of Theorem 6.1. We are given a solenoidal presentation $\mathcal{P}$ and a homeomorphism $\Phi_{\mathcal{P}}: \mathfrak{M} \rightarrow \mathcal{S}_{\mathcal{P}}$, and a presentation $\mathcal{Q}=\left\{q_{\ell+1}: M \rightarrow M \mid \ell \geq 0\right\}$, where each map $q_{\ell+1}$ is a continuous surjection, and a homeomorphism $\Phi_{\mathcal{Q}}: \mathfrak{M} \cong \mathcal{S}_{\mathcal{Q}}$. Let $\lambda_{\mathcal{F}}>0$ as in Lemma 6.3. chosen so that for all $x \in \mathfrak{M}, D_{\mathcal{F}}\left(x, \lambda_{\mathcal{F}}\right)$ is strongly convex. 
Let $\epsilon_{\mathcal{P}}>0$ be such that for all $\xi \in \mathfrak{M}$ then $B_{\mathcal{S}_{\mathcal{P}}}\left(\Phi_{\mathcal{P}}(\xi), \epsilon_{\mathcal{P}}\right) \subset \Phi_{\mathcal{P}}\left(B_{\mathfrak{M}}\left(\xi, \lambda_{\mathcal{F}} / 4\right)\right)$.

Let $\epsilon_{\mathcal{Q}}>0$ be such that for all $\xi \in \mathfrak{M}$ then $B_{\mathcal{S}_{\mathcal{Q}}}\left(\Phi_{\mathcal{Q}}(\xi), \epsilon_{\mathcal{Q}}\right) \subset \Phi_{\mathcal{Q}}\left(B_{\mathfrak{M}}\left(\xi, \lambda_{\mathcal{F}} / 4\right)\right)$.

Set $\epsilon_{\Phi} \equiv \min \left\{\epsilon_{\mathcal{P}}, \epsilon_{\mathcal{Q}}, \lambda_{M} / 4\right\}$, and let $\widehat{\epsilon} \equiv\left\{\epsilon_{0}>\epsilon_{1}>\epsilon_{2}>\cdots\right\}$ with $\lim _{\ell \rightarrow \infty} \epsilon_{\ell}=0$ and $\epsilon_{0}<\epsilon_{\Phi}$.

We are given the diagram (27), and the homeomorphism $\Phi=\mathcal{S}_{\mathcal{Q}} \circ \mathcal{S}_{\mathcal{P}}^{-1}: \mathcal{S}_{\mathcal{P}} \rightarrow \mathcal{S}_{\mathcal{Q}}$, for which

(1) the maps $\widehat{\lambda}=\left\{\lambda_{\ell}: M_{i_{\ell+1}} \rightarrow M \mid \ell \geq 0\right\}$ are continuous surjections which $\widehat{\epsilon}$-commute;

(2) the maps $\widehat{\mu}=\left\{\mu_{\ell}: M \rightarrow M_{i_{\ell}} \mid \ell \geq 0\right\}$ are covering maps which $\widehat{\epsilon}$-homotopy commute;

(3) $S(\widehat{\mu}): \mathcal{S}_{\mathcal{Q}} \rightarrow \mathcal{S}_{\mathcal{P}}$ is $\epsilon_{\Phi}$-homotopic to $\Phi^{-1}=\Phi=\mathcal{S}_{\mathcal{P}} \circ \mathcal{S}_{\mathcal{Q}}^{-1}$.

Choose a basepoint $z \in \mathfrak{M}$, then set $x=\Phi_{\mathcal{P}}(z)$ and $y=\Phi_{\mathcal{Q}}(z)$. We show that the squares in (46) below are commutative, and that the properties (37) and (38) are satisfied.

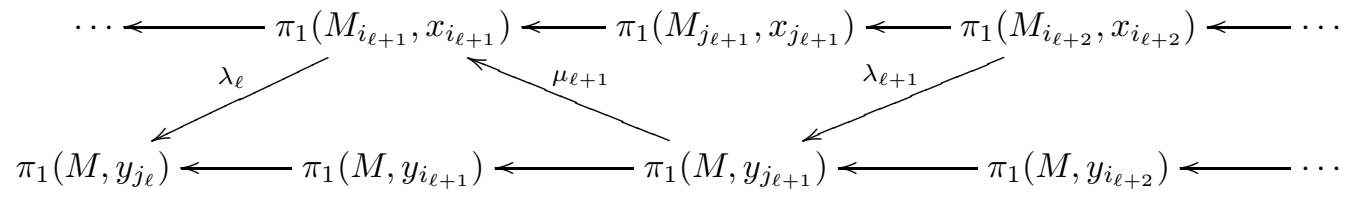

As noted before, that the maps $\widehat{\mu}$ induce commutative squares in the diagram (46).

We first show that the maps $\hat{\lambda}$ induce commutative squares in the diagram (46). Let $\alpha \in \pi_{1}\left(M_{i_{\ell+2}}, x_{i_{\ell+2}}\right)$ be represented by a closed smooth curve

$$
\gamma_{\alpha}:[0,1] \rightarrow M_{i_{\ell+2}} \quad, \quad \gamma_{\alpha}(0)=\gamma_{\alpha}(1)=x_{i_{\ell+2}} .
$$

Then $\lambda_{\ell} \circ p_{i_{\ell+2}}^{i_{\ell+1}}(\alpha) \in \pi_{1}\left(M, y_{j_{\ell}}\right)$ is represented by the closed curve $\lambda_{\ell} \circ p_{i_{\ell+2}}^{i_{\ell+1}} \circ \gamma_{\alpha}:[0,1] \rightarrow M$, and $q_{j_{\ell}}^{j_{\ell+1}} \circ \lambda_{\ell+1}(\alpha)$ is represented by the closed curve $q_{j_{\ell}}^{j_{\ell+1}} \circ \lambda_{\ell+1} \circ \gamma_{\alpha}:[0,1] \rightarrow M$. We claim these two curves are homotopic.

Let $\widetilde{\gamma_{\alpha}}:[0,1] \rightarrow L$ with $\widetilde{\gamma_{\alpha}}(0)=z$ be the lift of $\gamma_{\alpha}$ via the covering map $\Pi_{i_{\ell+2}}^{\mathcal{P}} \circ \Phi_{\mathcal{P}}: L \rightarrow M_{i_{\ell+2}}$.

Then $\Pi_{j_{\ell+1}}^{\mathcal{Q}} \circ \Phi_{\mathcal{Q}} \circ \widetilde{\gamma_{\alpha}}:[0,1] \rightarrow M$. It is given that the maps $\hat{\lambda}$ all $\widehat{\epsilon}$-commute, which by the definition (20) implies that

$$
d_{M}\left(\lambda_{\ell+1} \circ \Pi_{i_{\ell+2}}^{\mathcal{P}} \circ \Phi_{\mathcal{P}} \circ \widetilde{\gamma_{\alpha}}(t), \Pi_{j_{\ell+1}}^{\mathcal{Q}} \circ \Phi_{\mathcal{Q}} \circ \widetilde{\gamma_{\alpha}}(t)\right) \leq \epsilon_{j_{\ell+1}} 0 \leq t \leq 1
$$

As $\epsilon_{j_{\ell+1}} \leq \lambda_{M} / 4$ by the choice of $\epsilon_{\Phi}$ for each $0 \leq t \leq 1$, there is a geodesic segment between $\lambda_{\ell+1} \circ \Pi_{i_{\ell+2}}^{\mathcal{P}} \circ \Phi_{\mathcal{P}} \circ \widetilde{\gamma_{\alpha}}(t)$ and $\Pi_{j_{\ell+1}}^{\mathcal{Q}} \circ \Phi_{\mathcal{Q}} \circ \widetilde{\gamma_{\alpha}}(t)$. Thus, if we form a closed curve in $M$ by joining the endpoints of $\Pi_{j_{\ell+1}}^{\mathcal{Q}} \circ \Phi_{\mathcal{Q}} \circ \widetilde{\gamma_{\alpha}}$ to $y_{j_{\ell+1}}$ by a geodesic segments in $B_{M}\left(y_{j_{\ell+1}}, \lambda_{M} / 4\right)$, we obtain a curve which represents $\lambda_{\ell+1}(\alpha)$. A similar argument shows that

$$
\Pi_{j_{\ell}}^{\mathcal{Q}} \circ \Phi_{\mathcal{Q}} \circ \widetilde{\gamma_{\alpha}}=q_{j_{\ell+1}}^{j_{\ell}} \circ \Pi_{j_{\ell+1}}^{\mathcal{Q}} \circ \Phi_{\mathcal{Q}} \circ \widetilde{\gamma_{\alpha}}:[0,1] \rightarrow M
$$

represents the class $\lambda_{\ell} \circ p_{i_{\ell+2}}^{i_{\ell+1}}(\alpha) \in \pi_{1}\left(M, y_{j_{\ell}}\right)$, while the equality (48) implies that it also represents the class $q_{j_{\ell}}^{j_{\ell+1}} \circ \lambda_{\ell+1}(\alpha)$. Thus, they are equal as was to be shown.

We next show that the induced maps $\lambda_{\ell}$ and $\mu_{\ell}$ are monomorphisms. Let $\alpha \in \pi_{1}\left(M_{i_{\ell+1}}, x_{i_{\ell+1}}\right)$ and suppose that $\lambda_{\ell}(\alpha) \in \pi_{1}\left(M, y_{j_{\ell}}\right)$ is trivial. We claim this implies $\alpha$ is also trivial. As above, let $\alpha$ be represented by a closed smooth curve

$$
\gamma_{\alpha}:[0,1] \rightarrow M_{i_{\ell+1}} \quad, \quad \gamma_{\alpha}(0)=\gamma_{\alpha}(1)=x_{i_{\ell+1}},
$$

and let $\widetilde{\gamma_{\alpha}}:[0,1] \rightarrow L$ with $\widetilde{\gamma_{\alpha}}(0)=z$ be the lift of $\gamma_{\alpha}$ via the covering map $\Pi_{i_{\ell+1}}^{\mathcal{P}} \circ \Phi_{\mathcal{P}}: L \rightarrow M_{i_{\ell+1}}$. Then $\Pi_{j_{\ell}}^{\mathcal{Q}} \circ \Phi_{\mathcal{Q}} \circ \widetilde{\gamma_{\alpha}}:[0,1] \rightarrow M$ represents $\lambda_{\ell}(\alpha)$, where as before we obtain a closed curve by adjoining short geodesic segments to the endpoints. The assumption $\lambda_{\ell}(\alpha)$ is trivial class implies that $\lambda_{\ell} \circ \alpha:[0,1] \rightarrow M$ extends to a homotopy to the constant map, which is given by a map $H(t, s):[0,1]^{2} \rightarrow M$ with $H(t, 0)=\lambda_{\ell} \circ \alpha(t)$ and $H(t, 1)=H(0, s)=H(1, s)=y_{j_{\ell}}$ for all $0 \leq t, s \leq 1$. Then by Proposition 6.5 there is an approximate lift $\widehat{H}:[0,1]^{2} \rightarrow L$ such that $\Pi_{j_{\ell}}^{\mathcal{Q}} \circ \Phi_{\mathcal{Q}} \circ \widehat{H}$ is $\epsilon_{j_{\ell}}$ 
close to $H$, and hence is $\epsilon_{j_{\ell}}$-homotopic. Then the composition $\Pi_{i_{\ell+1}}^{\mathcal{P}} \circ \Phi_{\mathcal{P}} \circ \widehat{H}:[0,1]^{2} \rightarrow M_{i_{\ell+1}}$ defines a homotopy of $\alpha$ to an almost constant map, hence $\alpha$ is the trivial class, as was to be shown.

The claim that the maps $\mu_{\ell}$ induce injective maps on fundamental groups follows similarly.

The claim that the properties (37) and (38) are satisfied now follows in exactly the same way as in the proof of Theorem [5.1. This completes the proof of Theorem 6.1.

As remarked previously, Mardešić and Segal show in [57, Chapter II, Section 3.3] that the prohomotopy groups pro- $\pi_{k}(\mathfrak{M}, z)$ are well-defined for pointed shape expansions. The key technical fact used in this section, Proposition 6.5, applies equally well for the study of the higher pro-homotopy groups pro- $\pi_{k}(\mathfrak{M}, z)$ of a weak solenoid, for $k>1$. Instead of considering lifts of paths to the leaf $L$, one considers lifts of cubes. Then essentially the same arguments as above yield a proof of the following result:

THEOREM 6.6. Let $\mathfrak{M}$ be a matchbox manifold which is $M$-like. For $k>1$, pro- $\pi_{k}(\mathfrak{M}, z)$ is isomorphic to the pro-group defined by maps $\left\{g_{\ell}: \pi_{k}(M, y) \rightarrow \pi_{k}(M, y) \mid \ell \geq 0\right\}$. In particular, pro- $\pi_{k}(\mathfrak{M}, z)$ is independent of the choice of basepoint $z \in \mathfrak{M}$.

\section{Proofs of MAIN THEOREMS}

In this section, we show how the conclusions and proof of Theorem 6.1 imply Theorems 1.8 and 1.9 from the introduction. We assume that $\mathfrak{M}$ be an $n$-dimensional matchbox manifold which is $M$-like, for a closed manifold $M$. By Theorem 1.6, there exists a presentation $\mathcal{P}=\left\{f_{\ell+1}: M_{\ell+1} \rightarrow M_{\ell} \mid\right.$ $\ell \geq 0\}$ as a weak solenoid, and a homeomorphism $\Phi_{\mathcal{P}}: \mathfrak{M} \rightarrow \mathcal{S}_{\mathcal{P}}$. By Theorem 3.4, there exists a presentation $\mathcal{Q}=\left\{q_{\ell+1}: M \rightarrow M \mid \ell \geq 0\right\}$, where all the maps $q_{\ell+1}$ are continuous surjections, and a homeomorphism $\Phi_{\mathcal{Q}}: \mathfrak{M} \rightarrow \mathcal{S}_{\mathcal{Q}}$. Choose a basepoint $z \in \mathfrak{M}$ and set $x=\Phi_{\mathcal{P}}(z)$ and $y=\Phi_{\mathcal{Q}}(z)$. By the proof of Theorem 6.1, we can assume that all of the squares in the diagram (42) are commuting.

Define the sub-presentation $\mathcal{P}^{\prime}$ of $\mathcal{P}$ given by the maps

$$
\mathcal{P}^{\prime}=\left\{p_{i_{\ell+1}}^{i_{\ell}}: M_{i_{\ell+1}} \rightarrow M_{i_{\ell}} \mid \ell \geq 0\right\} .
$$

Then by the basic properties of inverse limits, there is a canonical homeomorphism $\Phi^{\prime}: \mathcal{S}_{\mathcal{P}^{\prime}} \rightarrow \mathcal{S}_{\mathcal{P}}$.

From the proof of Theorem 6.1, the maps $\lambda_{\ell}$ and $\mu_{\ell}$ in the diagram (42) are monomorphisms, for all $\ell \geq 1$. Thus, we have the extended commutative diagram of group monomorphisms:

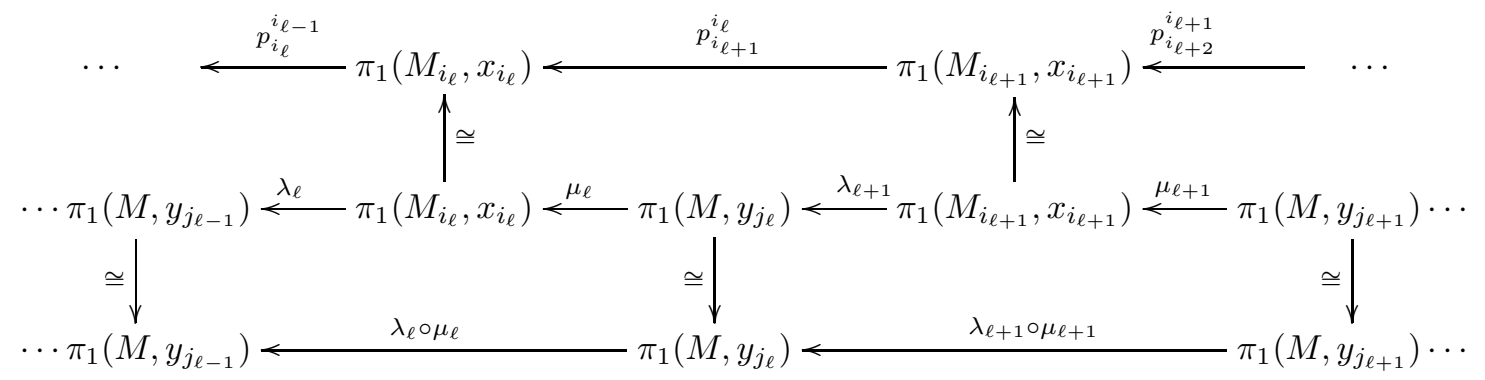

Recall that in (9) we defined $G_{\ell}=$ image $\left\{p_{\ell}^{0}: \pi_{1}\left(M_{\ell}, x_{\ell}\right) \longrightarrow G_{0}\right\}$, and the group chain $\mathcal{G}$ in (10) associated to the presentation $\mathcal{P}$ and basepoint $x_{0} \in M_{0}$. Define the group chain

$$
\mathcal{H}=H_{\ell}=\text { image }\left\{p_{\ell}^{0} \circ \mu_{\ell}: \pi_{1}\left(M, y_{j_{\ell}}\right) \longrightarrow G_{0}\right\},
$$

then (50) implies we then obtain a group chain

$$
G_{0} \supset G_{i_{1}} \supset H_{j_{1}} \supset G_{i_{2}} \supset \cdots \supset G_{i_{\ell}} \supset H_{j_{\ell}} \supset G_{i_{\ell+1}} \supset H_{j_{\ell+1}} \supset \cdots .
$$

Set $H=\pi_{1}\left(M, y_{0}\right)$. As $M$ is connected, for all $\ell>0$ there is an isomorphism $H_{\ell} \cong H$. 
Now define $N_{\ell}$ to be the covering space of $M_{0}$ associated to the subgroup $H_{\ell}$. The chain of subgroups in (52) then yields a presentation $\mathcal{P}^{\prime}=\left\{q_{\ell+1}: N_{\ell+1} \rightarrow N_{\ell} \mid \ell \geq 1\right\}$. By Theorem 2.7, the spaces $\mathcal{S}_{\mathcal{P}}$ and $\mathcal{S}_{\mathcal{P}^{\prime}}$ are homeomorphic. This completes the proof of Theorem 1.8

For the proof of Theorem 1.9, recall that $M$ aspherical means that $\pi_{k}\left(M, y_{0}\right)=\{0\}$ is the trivial group for all $k>1$ and choice of basepoint $y_{0}$. The key technical fact used in the proof of Theorem 6.1, the approximate homotopy lifting property in Proposition 6.5, applies equally well for the study of the maps between higher homotopy groups in diagram (39). Instead of considering lifts of paths to the leaf $L \subset \mathfrak{M}$, one considers lifts of cubes. We require only the conclusion that the following diagram commutes, for each $k>1$ and $\ell \geq 1$ :

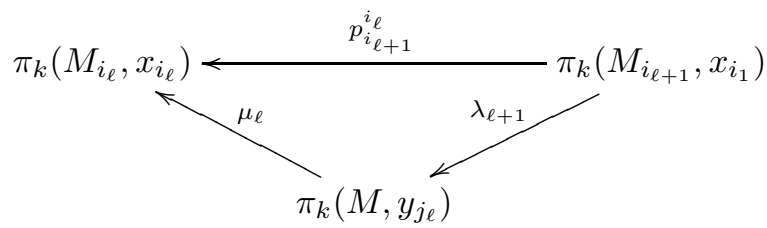

The covering map $p_{\ell+1}: M_{\ell+1} \rightarrow M_{\ell}$ induces an isomorphism on homotopy groups for $k>1$, hence the horizontal map $p_{i_{\ell+1}}^{i_{\ell}}$ in (53) is an isomorphism. Thus, $\pi_{k}\left(M_{i_{\ell}}, x_{i_{\ell}}\right)=\{0\}$ is trivial for all $\ell \geq 1$, and also $\pi_{k}\left(N_{\ell}, n_{\ell}\right)=\{0\}$ is trivial for the covering space $N_{\ell} \rightarrow M_{i_{\ell}}$ defined above, where $n_{\ell} \in N_{\ell}$ is some lift of $x_{i_{\ell}}$. Hence, each $N_{\ell}$ is an aspherical space. Thus, the lift of $\mu_{\ell}$ to a map $h_{\ell}: M \rightarrow N_{\ell}$ induces isomorphisms on all homotopy groups, so is a homotopy equivalence. By the assumption that $M$ satisfies the Borel Conjecture, it follows that $h_{\ell}$ is homotopic to a homeomorphism $f_{\ell}$ for each $\ell \geq 1$. Using the homeomorphisms $\left\{f_{\ell}\right\}$ we obtain a presentation $\mathcal{P}^{\prime \prime}=\left\{f_{\ell}^{-1} \circ q_{\ell+1} \circ f_{\ell+1}: M \rightarrow M \mid \ell \geq 1\right\}$ for which $\mathcal{S}_{\mathcal{P}^{\prime \prime}}$ is homeomorphic to $\mathcal{S}_{\mathcal{P}^{\prime}}$ and hence to $\mathcal{S}_{\mathcal{P}}$, which shows the claim of Theorem 1.9.

\section{EXAMPLES}

In this section, we give a collection of examples, some from the literature and some novel, which illustrate the conclusions of Theorems 1.8 and 1.9. These examples are based on the basic observation of this work, that the $M$-like hypothesis on a solenoid is a shape version of the non co-Hopfian property for manifolds.

Recall that a group $G$ is co-Hopfian if there does not exist an embedding of $G$ to a proper subgroup of itself with finite index, and non co-Hopfian otherwise. (In some literature, a group is said to be co-Hopfian if every embedding of $G$ to a proper subgroup of itself must be an isomorphism, and they refer to our definition as the finitely co-Hopfian property. By non co-Hopfian, we will always mean finitely non co-Hopfian.) The co-Hopfian concept for groups was first studied by Baer in [6], where they are referred to as "S-groups".

A closed connected manifold $M$ is co-Hopfian if every finite covering map $\pi: M \rightarrow M$ is a homeomorphism, and non co-Hopfian otherwise. Given a proper self-covering map $\pi: M \rightarrow M$, choose a basepoint $x_{1} \in M$ and set $x_{0}=\pi\left(x_{1}\right)$. Then $G_{0}=\pi_{1}\left(M, x_{0}\right)$ is a non co-Hopfian group.

Given a non co-Hopfian manifold $M$ with proper self-covering map $\pi: M \rightarrow M$, we obtain a presentation $\mathcal{P}=\left\{p_{\ell+1}: M_{\ell+1} \rightarrow M_{\ell} \mid \ell \geq 0\right\}$, where each $M_{\ell}=M$ and $p_{\ell+1}=\pi$. As remarked in Section 1 the associated weak solenoid $\mathcal{S}_{\mathcal{P}}$ is always $M$-like.

Without additional hypotheses on $M$, the property that $G_{0}=\pi_{1}\left(M, x_{0}\right)$ is a non co-Hopfian group does not imply that $M$ is a non co-Hopfian manifold. Here is a simple construction to show that. Let $B$ be a non co-Hopfian manifold of dimension $m \geq 4$. Let $M_{0}$ be the result of attaching the product space $\mathbb{S}^{2} \times \mathbb{S}^{m-2}$ to $B$ along the boundary of a disk in each manifold. Then the fundamental group of $M_{0}$ equals that of $B$. Note that the second homotopy group $\pi_{2}\left(M_{0}, x_{0}\right)$ contains a free abelian summand corresponding to the attached copy of $\mathbb{S}^{2}$. For a proper covering $\pi: M_{1} \rightarrow M_{0}$, the rank of $\pi_{2}\left(M_{1}, x_{1}\right)$ is then greater than that of $M_{0}$, hence they cannot be homeomorphic. 
This construction illustrates the requirement that the base manifold $M_{0}$ be aspherical in Theorem 1.9 . The additional requirement that the coverings of $M_{0}$ also satisfy the Borel Conjecture is made so that all coverings of $M_{0}$ are then homeomorphic to $M_{0}$. Thus, a closed aspherical manifold $M$ which satisfies the strongly Borel property [18, Definition 1.4] is co-Hopfian if and only if its fundamental group is co-Hopfian.

The non co-Hopfian property was also used in the work of Biś, Hurder and Shive [10, Section 3] to construct many new classes of foliations which generalize the original construction of Hirsch in 46] that was based on the construction of the Smale solenoid by self-embeddings of a solid torus.

We next recall some examples of weak solenoids obtained by explicit constructions of non co-Hopfian closed manifolds, and then discuss some examples obtained from more abstract methods.

8.1. Abelian group chains. Let $G_{0}=\mathbb{Z}^{k}$ for $k \geq 1$ be the free abelian group of rank $k$, with basis vectors $\left\{\vec{e}_{1}, \ldots, \vec{e}_{k}\right\}$. Choose any collection of vectors $\left\{\vec{v}_{1}, \ldots, \vec{v}_{k}\right\} \subset \mathbb{Z}^{k}$ which are linearly independent over $\mathbb{Q}$. Then define $\phi: \mathbb{Z}^{k} \rightarrow \mathbb{Z}^{k}$ by setting $\phi\left(\vec{e}_{i}\right)=\vec{v}_{i}$ for $1 \leq i \leq k$. The map $\phi$ is an endomorphism of $\mathbb{Z}^{k}$, and for most choices we obtain a proper embedding. We obtain a group chain by setting $\mathcal{G}_{\phi}=\left\{G_{\ell} \equiv \phi^{\ell}\left(G_{0}\right) \mid \ell \geq 0\right\}$.

We illustrate this with the simplest example, where $k=1$. Choose distinct primes $p, q \geq 2$. Define $G_{\ell}=\left\{n \cdot p^{\ell} \mid n \in \mathbb{Z}\right\}$ and $H_{\ell}=\left\{n \cdot q^{\ell} \mid n \in \mathbb{Z}\right\}$, so we obtain group chains defined by the selfembeddings, $\mathcal{P}=\left\{G_{\ell} \mid \ell \geq 1\right\}$ and $\mathcal{Q}=\left\{H_{\ell} \mid \ell \geq 1\right\}$. Criteria for the equivalence of group chains in $\mathbb{Z}$ were given by Bing [9], McCord [59, Aarts and Fokkink [2], as well as in [18, Section 5]. In particular, $\mathcal{P}$ and $\mathcal{Q}$ are not return equivalent, so we obtain the well-known fact that the solenoids $\mathcal{S}_{\mathcal{P}}$ and $\mathcal{S}_{\mathcal{Q}}$ are not homeomorphic.

More generally, every group chain $\mathcal{G}$ in $\mathbb{Z}$ defines a scale in the sense of 63 , see also the discussion in Section 8.7. On the other hand, a monomorphism $\phi: \mathbb{Z} \rightarrow \mathbb{Z}$ is determined by the integer $m=\phi(1)$, which has a finite number of prime divisors, where $m=q_{1} \cdots q_{k}$ with each $q_{i}$ prime though possibly not distinct. As an example, let $\left\{p_{1}<p_{2}<\cdots\right\}$ be an infinite collection of increasing primes. Define $K_{\ell}=\left\{n \cdot p_{1} p_{2} \cdots p_{\ell} \mid n \in \mathbb{Z}\right\} \cong \mathbb{Z}$ and let $\mathcal{K}=\left\{K_{\ell} \mid \ell \geq 0\right\}$ be the corresponding group chain. Then the group chain $\mathcal{K}$ is not return equivalent to any group chain defined by a monomorphism $\phi: \mathbb{Z} \rightarrow \mathbb{Z}$, and thus the weak solenoid $\mathcal{S}_{\mathcal{K}}$ is not homeomorphic to $\mathcal{S}\left(\mathcal{G}_{\phi}\right)$.

As a consequence, we see that there are uncountably many circle-like weak solenoids which are distinct up to homeomorphism, and not homeomorphic to the solenoid obtained from a covering map $\pi: \mathbb{S}^{1} \rightarrow \mathbb{S}^{1}$. Thus, the conclusion of Theorem 1.9 cannot be strengthened in general.

8.2. Coverings of the Klein bottle. This example is a generalization to arbitrary integer $d>1$ of the example due to Rogers and Tollefson [69, see also Fokkink and Oversteegen [38]. Consider a map of the plane, given by a translation by $\frac{1}{2}$ in the first component, and by reflection in the second component, i.e.

$$
r \times i: \mathbb{R}^{2} \rightarrow \mathbb{R}^{2} \text { where }(x, y) \mapsto\left(x+\frac{1}{2},-y\right) .
$$

This map commutes with translations by the elements in the integer lattice $\mathbb{Z}^{2} \subset \mathbb{R}^{2}$, and so induces the map $r \times i: \mathbb{T}^{2}=\mathbb{R}^{2} / \mathbb{Z}^{2} \rightarrow \mathbb{T}^{2}$ of the torus. This map is an involution, and the quotient space $K=\mathbb{T}^{2} /(x, y) \sim r \times i(x, y)$ is homeomorphic to the Klein bottle.

Let $L: \mathbb{T}^{2} \rightarrow \mathbb{T}^{2}$ be the $d$-fold covering map given by $L(x, y)=(x, d y)$, and form the inverse limit $\mathbb{T}_{\infty}=\lim _{\longleftarrow}\left\{L: \mathbb{T}^{2} \rightarrow \mathbb{T}^{2}\right\}$, which is a solenoid with 2 -dimensional leaves. Let $x_{0}=(0,0) \in M_{0}=\mathbb{T}^{2}$. The fundamental group $G_{0}=\mathbb{Z}^{2}$ is abelian, so for any $x, y \in \mathfrak{X}_{0}$ the kernels $K\left(\mathcal{G}^{x}\right)=K\left(\mathcal{G}^{y}\right) \cong \mathbb{Z}$, and every leaf is homeomorphic to an open two-ended cylinder.

The involution $r \times i$ is compatible with the covering maps $L$, and so it induces an involution $(r \times$ $i)_{\infty}: \mathbb{T}_{\infty} \rightarrow \mathbb{T}_{\infty}$, which is seen to have a single fixed point $(0,0, \ldots) \in \mathbb{T}_{\infty}$, and permutes the other path-connected components. Let $p: K \rightarrow K$ be the $d$-fold covering of the Klein bottle by itself, given by $p(x, y)=(x, d y)$, and consider the inverse limit space $K_{\infty}=\underset{\longleftarrow}{\lim }\{p: K \rightarrow K\}$. Note that taking the quotient by the involution $r \times i$ is compatible with the covering maps $L$ and $p$; that is, 
$p \circ(r \times i)=L$, and so induces the map $i_{\infty}: \mathbb{T}_{\infty} \rightarrow K_{\infty}$ of the inverse limit spaces. Under this map, the path-connected component of the fixed point $(0,0, \ldots)$ is identified so as to become a nonorientable one-ended cylinder. The image of any other path-connected component is an orientable 2-ended cylinder, so the space $K_{\infty}$ is not homogeneous.

Let $x=\left(x_{\ell}\right) \in K_{\infty}$ for $x_{\ell} \in K$. Then $G_{0}=\pi_{1}\left(K, x_{0}\right)=\left\langle a, b \mid b a b^{-1}=a^{-1}\right\rangle$. The group chain associated to this solenoid is given by the subgroups $G_{\ell}=\left\langle a^{d^{\ell}}, b\right\rangle \subset G_{0}$. For the group chain $\mathcal{G}^{x}$, we have that the kernel $K\left(\mathcal{G}^{x}\right)=\langle b\rangle$. If a point $y \in K_{\infty}$ is in a leaf which is orientable, then the kernel of the group chain $\mathcal{G}^{y}=\left\langle b^{2}\right\rangle$. If $y \in K_{\infty}$ is in the non-orientable leaf, then the kernel $K\left(\mathcal{G}^{y}\right)$ is conjugate to $K\left(\mathcal{G}^{x}\right)$.

It follows that the effective action of $G_{0}$ on the fiber of the solenoid is that of the dihedral group $\left\langle a, b \mid b a b^{-1}=a^{-1}, b^{2}=1\right\rangle$. This action is that of the iterated monodromy group associated to a Chebyshev polynomial of degree $d$, as discussed in [54].

8.3. Heisenberg group chains. Let $\mathcal{H}$ be the 3 -dimensional Heisenberg Lie group, and let $G_{0}=\mathbb{H}$ be the discrete Heisenberg group. These groups are presented as the upper-triangular subgroup of the $3 \times 3$ matrices:

$$
\mathcal{H} \equiv\left(\begin{array}{ccc}
1 & x & z \\
0 & 1 & y \\
0 & 0 & 1
\end{array}\right), x, y, z \in \mathbb{R} \quad, \quad \mathbb{H} \equiv\left(\begin{array}{ccc}
1 & a & c \\
0 & 1 & b \\
0 & 0 & 1
\end{array}\right), a, b, c \in \mathbb{Z}
$$

The group $\mathcal{H}$ can also be presented in the form $\left\{\mathbb{R}^{3}, *\right\}$, with the group operation $*$ given by $(x, y, z) *\left(x^{\prime}, y^{\prime}, z^{\prime}\right)=\left(x+x^{\prime}, y+y^{\prime}, z+z^{\prime}+x y^{\prime}\right)$. The group $\mathbb{H}$ is the simplest example of a torsion-free nilpotent group which is not abelian.

Wouter Van Limbeek suggested to the authors the following simple construction of a self-embedding of $G_{0}$, which yields a group chain that is not normal. Define $\phi: G_{0} \rightarrow G_{0}$ given by $\phi(a, b, c)=$ $(2 a, 2 b, 4 c)$, which yields a group monomorphism. Let

$$
G_{\ell}=\phi^{\ell}\left(G_{0}\right)=\left\{\left(2^{\ell} a, 2^{\ell} b, 4^{\ell} c\right) \mid a, b, c \in \mathbb{Z}\right\}
$$

It is then an exercise to check that the normal core of $G_{\ell}$ is the subgroup

$$
C_{\ell}=\left\{\left(4^{\ell} a, 4^{\ell} b, 4^{\ell} c\right) \mid a, b, c \in \mathbb{Z}\right\} \subset G_{\ell} .
$$

Note that $G_{2 \ell} \subset C_{\ell}$ so the group chain $\left\{G_{\ell} \mid \ell \geq 0\right\}$ is equivalent to a normal chain, hence the solenoid defined by self-coverings of $M_{0}=\mathcal{H} / G_{0}$ will be homogeneous by [38, and $M$-like by construction.

On the other hand, Jessica Dyer gave in her thesis [30] examples of group chains in $G_{0} \subset \mathbb{H}$ for which the discriminant of the Cantor action constructed from the chain is a Cantor group. We recall Example 8.5 from [31. Choose distinct primes $p>1$ and $q>1$, and define

$$
H_{\ell}=\left\{\left(p^{\ell} a, q^{\ell} b, p^{\ell} c\right) \mid a, b, c \in \mathbb{Z}\right\} .
$$

Note that $H_{1}$ is the image of the set map $\phi(a, b, c)=(p a, q b, p c)$, but this $\phi$ is not a homomorphism for the group law on $\mathbb{H}$. On the other hand, it is shown in 31 that the solenoid defined by the associated coverings $M_{\ell}=\mathcal{H} / H_{\ell}$ of $M_{0}=\mathcal{H} / \mathbb{H}$ is not homogeneous.

The following is an interesting open problem:

PROBLEM 8.1. Let $G_{0} \subset \mathbb{H}$ be as above. Does there exists a subgroup $G_{1} \subset G_{0}$ of finite index, such that for $M=\mathcal{H} / G_{1}$, there exists an $M$-like weak solenoid which is not homogeneous?

In terms of group chains, does there exists $G_{1} \subset G_{0}$ of finite index, and a proper self-embedding $\phi: G_{1} \rightarrow G_{1}$, such that the associated group chain $\mathcal{G}(\phi) \equiv\left\{G_{\ell}=\phi^{\ell}\left(G_{1}\right) \mid \ell \geq 1\right\}$ has trivial kernel, and the associated weak solenoid is not homogeneous? 
8.4. Nil-manifolds. There are many generalizations of the above constructions of weak solenoids obtained from lattices in the Heisenberg group. The Heisenberg group $\mathcal{H}$ is replaced with any connected nilpotent Lie group $\mathcal{N}$, and $\mathbb{H}$ is replaced with a cocompact lattice subgroup $\Gamma \subset \mathcal{N}$. For example, Belegradek considered in [8] when such a lattice must be co-Hopfian, and in particular when they are not. Non co-Hopfian subgroups of nilpotent Lie groups were also studied by Dekimpe, Lee and Potyagailo in 22, 23, 24, and by Cornulier in [20. Here is a general version of Problem 8.1

PROBLEM 8.2. Let $\mathcal{N}$ be a connected Lie group. Does there exists a lattice subgroup $\Gamma \subset \mathcal{N}$ which admits a proper self-embedding $\phi: \Gamma \rightarrow \Gamma$, such that the group chain $\mathcal{G}(\phi) \equiv\left\{G_{\ell}=\phi^{\ell}(\Gamma) \mid \ell \geq 0\right\}$ has trivial kernel, and the associated weak solenoid is not homogeneous.

This problem is closely related to the works of Reid [65, Willis [81] and Van Limbeek in [76, 77, on the properties of profinite groups derived from a self-embedding of a non co-Hopfian group, in terms of contracting maps for profinite groups.

8.5. Non co-Hopfian 3-manifolds. The question of which compact 3-manifolds admit proper self-coverings has been studied in detail by González-Acuña, Litherland and Whitten in the works 44 and [45], and see also the work [80] by Wang and Wu.

8.6. Higher dimensional constructions. Note that the product of any finite collection of non coHopfian manifolds is again non co-Hopfian. Thus, we can form the products of an arbitrary collection of examples as given above to obtain a non co-Hopfian manifold of arbitrarily large dimension.

A more general approach is to consider the problem of which finitely-presented groups $G_{0}$ are non co-Hopfian? One must then also ask, when such a group can be realized as the fundamental group of a non co-Hopfian manifold? Such constructions usually result in a base manifold of dimension $m \geq 4$. We recall some results in the literature on when a group is non co-Hopfian.

Endimioni and Robinson give in [35] some sufficient conditions for a group to be non co-Hopfian. Delgado and Timm consider in [25] the co-Hopfian condition for the fundamental groups of connected finite complexes, which is certainly a requirement for the construction of non co-Hopfian manifolds.

Ohshika and Potyagailo gave example in [64] of a freely indecomposable geometrically finite torsionfree non-elementary Kleinian group which is non co-Hopfian. Delzant and Potyagailo study in [26] non-elementary geometrically finite Kleinian groups are non co-Hopfian, and Kapovich and Weiss considered the co-Hopf property for word hyperbolic groups in [50.

On the other hand, the following problem appears to be open:

PROBLEM 8.3. Let $G_{0}$ be a finitely-generated non co-Hopfian group. Find conditions on $G_{0}$ so that it is realized as the fundamental group of a non co-Hopfian closed manifold $M_{0}$.

8.7. Scale-invariant groups. A finitely generated infinite group $G$ is called scale-invariant by Nekrashevych and Pete [63, if there is a group chain $\mathcal{G}=\left\{G_{\ell} \mid \ell \geq 0\right\}$ that each $G_{\ell}$ is isomorphic to $G$, and whose kernel $K(\mathcal{G})=\cap_{\ell \geq 0} G_{\ell}$ is a finite group. The assumption that the kernel is finite is a fundamental aspect of this concept.

Remark 2.4 identifies $K(\mathcal{G})$ with the fundamental group of the leaf $L_{x}$ containing the basepoint $x \in \mathfrak{M}$ used to define the group chain. Thus, if $\mathfrak{M}$ is an $M$-like matchbox manifold and $\mathcal{F}_{\mathfrak{M}}$ admits a leaf with finite fundamental group, Theorem 1.8 implies that the fundamental group $G_{0}=\pi_{1}(M, b)$ is scale-invariant.

A non co-Hopfian group $G_{0}$ with proper embedding $\phi: G_{0} \rightarrow G_{0}$ is scale-invariant if the intersection $\cap_{\ell \geq 0} \phi^{\ell}\left(G_{0}\right)$ is a finite group. Nekrashevych and Pete addressed in 63. the problem whether there exists scale invariant groups which are non co-Hopfian. In particular, they gave examples of scale invariant but non co-Hopfian groups, obtained from a cross-product of groups, some of which are nilpotent, and some are not. We briefly recall a special case of this construction. 
Fix $n \geq 1$ and let $G \subset \mathrm{GL}(n, \mathbb{Z})$. Define

$$
H=\left\{(\vec{n}, g) \mid \vec{n} \in \mathbb{Z}^{n}, g \in G\right\}
$$

where the product is defined by $\left(\vec{n}_{1}, g_{1}\right) \star\left(\vec{n}_{2}, g_{2}\right)=\left(\vec{n}_{1}+g_{1} \cdot \vec{n}_{2}, g_{1} g_{2}\right)$. Then $H$ is nilpotent if and only if the subgroup $G$ is nilpotent. Choose $p>1$ and define the monomorphism $\phi\left(\vec{n}_{1}, g_{1}\right)=\left(p \cdot \vec{n}_{1}, g_{1}\right)$. The Rogers and Tollefson Example 8.2 is the simplest example of this construction.

Suppose that $G=\pi_{1}(B, b)$ for a closed manifold $B$ which satisfies the strong Borel conjecture. Then let $M$ be the closed manifold which fibers over $B$, with fiber $\mathbb{T}^{n}$ twisted by the action of $G$. Then $M$ is again aspherical, and satisfies the strong Borel Conjecture. For example, we can take $G$ to be a group which acts freely on $\mathbb{R}^{m}$ by isometries and let $B=\mathbb{R}^{m} / G$ be the quotient. Then $M$ is a non co-Hopfian manifold, and the weak solenoid determined by the map $\phi$ is $M$-like. There are many variations on this construction.

\section{REFERENCES}

[1] J.M. Aarts and M. Martens, Flows on one-dimensional spaces, Fund. Math., 131:39-58, 1988.

[2] J.M. Aarts and R.J. Fokkink, The classification of solenoids, Proc. Amer. Math. Soc., 111 :1161-1163, 1991.

[3] J. Aarts and L. Oversteegen, Flowbox manifolds, Trans. Amer. Math. Soc., 327:449-463, 1991.

[4] J. Aarts and L. Oversteegen, Matchbox manifolds, In Continua (Cincinnati, OH, 1994), Lecture Notes in Pure and Appl. Math., Vol. 170, Dekker, New York, 1995, pages 3-14..

[5] P. Alexandroff, Untersuchungen über Gestalt und Lage abgeschlossener Mengen beliebiger Dimension, Ann. of Math., 30:101-187, 1928/29.

[6] R. Baer, Groups without proper isomorphic quotient groups, Bull. Amer. Math. Soc., 50:267-278, 1944.

[7] M. Barge and J. Kennedy, Continuum theory and topological dynamics, in Open problems in topology, North-Holland, Amsterdam, 1990, pages 633-644.

[8] I. Belegradek, On co-Hopfian nilpotent groups, Bull. London Math. Soc., 35:805-811, 2003.

[9] R.H. Bing, A simple closed curve is the only homogeneous bounded plane continuum that contains an arc, Canad. J. Math., 12:209-230, 1960.

[10] A. Biś, S. Hurder, and J. Shive, Hirsch foliations in codimension greater than one, In Foliations 2005, World Scientific Publishing Co. Inc., River Edge, N.J., 2006: 71-108.

[11] R. Bishop and R. Crittenden, Geometry of manifolds, (reprint of the 1964 original), AMS Chelsea Publishing, Providence, RI, 2001.

[12] A. K. Bousfield and D. M. Kan, Homotopy limits, completions and localizations, Lect. Notes in Math., 304, Springer-Verlag, 1972.

[13] A. Candel and L. Conlon, Foliations I, Amer. Math. Soc., Providence, RI, 2000.

[14] T.A. Chapman and S. Ferry, Approximating homotopy equivalences by homeomorphisms, Amer. J. Math., 101:583-607, 1979.

[15] A. Clark and S. Hurder, Embedding solenoids in foliations, Topology Appl., 158:1249-1270, 2011.

[16] A. Clark and S. Hurder, Homogeneous matchbox manifolds, Trans. Amer. Math. Soc., 365:3151-3191, 2013.

[17] A. Clark, S. Hurder and O. Lukina, Shape of matchbox manifolds, Indag. Math., 25(4):669-712, 2014.

[18] A. Clark, S. Hurder and O. Lukina, Classifying matchbox manifolds, Geom \& Top, to appear, arXiv:1311.0226.

[19] A. Clark, S. Hurder and O. Lukina, Manifold-like matchbox manifolds, Trans. A.M.S., to appear; arXiv:1704.04402.

[20] Y. Cornulier, Gradings on Lie algebras, systolic growth, and cohopfian properties of nilpotent groups, Bull. Soc. Math. France, 144:693-744, 2016.

[21] J.F. Davis, The work of Tom Farrell and Lowell Jones in topology and geometry, Pure Appl. Math. Q., $8: 1-14,2012$.

[22] K. Dekimpe and K.-B. Lee, Expanding maps on infra-nilmanifolds of homogeneous type, Trans. Amer. Math. Soc., 355:1067-1077, 2003.

[23] K. Dekimpe and K.-B. Lee, Expanding maps, Anosov diffeomorphisms and affine structures on infra-nilmanifolds, Topology Appl., 130:259-269, 2003.

[24] T. Dekimpe and L. Potyagailo, Expanding maps and non-trivial self-covers on infra-nilmanifolds, Topol. Methods Nonlinear Anal., 47:347-368, 2016.

[25] A.L. Delgado and M. Timm, Spaces whose finite sheeted covers are homeomorphic to a fixed space, Topology Appl., 129:1-10, 2003.

[26] T. Delzant and L. Potyagailo, Endomorphisms of Kleinian groups, Geom. Funct. Anal., 13:396-436, 2003.

[27] M. do Carmo, Riemannian geometry, Translated from the second Portuguese edition by Francis Flaherty, Birkhäuser Boston Inc., Boston, 1992.

[28] J. Dydak, Some remarks on the shape of decomposition spaces, Bull. Acad. Polon. Sci. Sér. Sci. Math. Astronom. Phys., 23:561-564, 1975.

[29] J. Dydak, Pointed and unpointed shape and pro-homotopy, Fund. Math. 107:57-69, 1980.

[30] J. Dyer, Dynamics of equicontinuous group actions on Cantor sets, doctoral dissertation, University of Illinois at Chicago, 2015. 
[31] J. Dyer, S. Hurder and O. Lukina, The discriminant invariant of Cantor group actions, Topology Appl., 208: 64-92, 2016.

[32] J. Dyer, S. Hurder and O. Lukina, Growth and homogeneity of matchbox manifolds, Indagationes Math., 28:145-169, 2017

[33] J. Dyer, S. Hurder and O. Lukina, Molino theory for matchbox manifolds, Pacific J. Math., 289:91-151, 2017.

[34] K. Eda, J. Mandić and V. Matijević, Torus-like continua which are not self-covering spaces, Topology Appl., 153:359-369, 2005.

[35] G. Endimioni and D. Robinson, On co-Hopfian groups, Publ. Math. Debrecen, 67:423-436, 2005.

[36] S. Eilenberg, Sur les transformations à petite tranches, Fund. Math., 30:92-95, 1938.

[37] S. Ferry, Homotoping ع-maps to homeomorphisms, Amer. J. Math., 101:567-582, 1979.

[38] R. Fokkink and L. Oversteegen, Homogeneous weak solenoids, Trans. Amer. Math. Soc., 354(9):3743-3755, 2002.

[39] M.K. Fort, Jr. and M.C. McCord, Approximation of maps of inverse limit spaces by induced maps, Fund. Math., 59:323-329, 1966

[40] R. Fox, On shape, Fund. Math., 74:47-71, 1972.

[41] H. Freudenthal, Entwicklungen von Räumen und Gruppen, Rec. Math. Moscou, N. Sr. 1:677-681, 1936. Available in Hans Freudenthal selecta, Heritage of European Mathematics, Edited by Tonny A. Springer and Dirk van Dalen, European Mathematical Society (EMS), Zürich, 2009.

[42] T. Ganea, On E-maps onto manifolds, Fund. Math. 47:35-44, 1959.

[43] É Ghys, Laminations par surfaces de Riemann, in Dynamique et Géométrie Complexes, Panoramas \& Synthèses, 8:49-95, 1999

[44] F. González-Acuña and R. Litherland and W. Whitten, Co-Hopficity of Seifert-bundle groups, Trans. Amer. Math. Soc., 341:143-155, 1994.

[45] F. González-Acuña and W. Whitten, Co-Hopficity of 3-manifold groups, Topology Appl., 56:87-97, 1994.

[46] M. Hirsch, A stable analytic foliation with only exceptional minimal sets, in Dynamical Systems, Warwick, 1974, Lect. Notes in Math. vol. 468, , Springer-Verlag, 1975, 9-10.

[47] S. Hurder, Lectures on Foliation Dynamics: Barcelona 2010, Proceedings of Conference on Geometry and Topology of Foliations (C.R.M. 2010), 2014.

[48] S. Hurder and O. Lukina, Wild solenoids, Transactions A.M.S., 2017, DOI: https://doi.org/10.1090/tran/7339.

[49] S. Hurder and O. Lukina, Orbit equivalence and classification of weak solenoids, submitted, 2018, arXiv:1803.02098

[50] I. Kapovich and D. Wise, On the failure of the co-Hopf property for subgroups of word-hyperbolic groups, Israel J. Math., 122:125-147, 2001.

[51] S.K. Kaul, Realization of mappings as inverse limits, Colloq. Math., 17:297-307, 1967.

[52] W. Lück, Aspherical manifolds, Bulletin of the Manifold Atlas, 1-17, 2012. http://131.220.77.52/lueck/publications.php\#survey

[53] O. Lukina, Arboreal Cantor actions, Journal L.M.S., 2018, arXiv:1801.01440

[54] O. Lukina, Galois groups and Cantor actions, preprint, 2018, arXiv:1809.08475

[55] M. Lyubich and Y. Minsky, Laminations in holomorphic dynamics, J. Differential Geom., 47:17-94, 1997.

[56] S. Mardešić and J. Segal, e-mappings onto polyhedra, Trans. Amer. Math. Soc., 109:146-164, 1963.

[57] S. Mardešić and J. Segal, Shape theory. The inverse system approach, North-Holland Mathematical Library, 26 North-Holland Publishing Co., Amsterdam-New York, 1982.

[58] S. Mardešić and V. Matijević, Classifying overlay structures of topological spaces, Topology Appl., 113:167-209, 2001.

[59] M.C. McCord, Inverse limit sequences with covering maps, Trans. Amer. Math. Soc., 114:197-209, 1965

[60] J. Mioduszewski, Mappings of inverse limits, Coll. Math. 10:39-44, 1963.

[61] C.C. Moore and C. Schochet, Analysis on Foliated Spaces, Math. Sci. Res. Inst. Publ. vol. 9, Second Edition, Cambridge University Press, New York, 2006.

[62] S. Nadler, Continuum theory, Monographs and Textbooks in Pure and Applied Mathematics, Vol. 158, Marcel Dekker, Inc., New York, 1992.

[63] V. Nekrashevych and G. Pete, Scale-invariant groups, Groups Geom. Dyn. 5:139-167, 2011.

[64] K. Ohshika and L. Potyagailo, Self-embeddings of Kleinian groups, Ann. Sci. École Norm. Sup. (4), 31:329 343, 1998

[65] C.D. Reid, Endomorphisms of profinite groups, Groups Geom. Dyn. 8:553-564, 2014.

[66] J.T. Rogers, Jr., Inverse limits of manifolds with covering maps, in Topology Conference (Proc. General Topology Conf., Emory Univ., Atlanta, Ga., 1970), Dept. Math., Emory Univ., Atlanta, Ga., 1970, pages $81-85$.

[67] J.T. Rogers, Jr. and J. L. Tollefson, Maps between weak solenoidal spaces, Coll. Math. 23(2):245-249, 1971.

[68] J.T. Rogers, Jr. and J.L. Tollefson, Homogeneous inverse limit spaces with non-regular covering maps as bonding maps, Proc. A.M.S, 29: 417-420, 1971.

[69] J.T. Rogers, Jr. and J.L. Tollefson, Involutions on solenoidal spaces, Fund. Math., 73:11-19, 1971/72.

[70] J.T. Rogers, Jr. and J. L. Tollefson, Homeomorphisms homotopic to induced homeomorphisms of weak solenoidal spaces, Coll. Math., 25:81-87, 1972.

[71] J.W. Rogers, Jr., Inducing approximations homotopic to maps between inverse limits, Fund. Math., 78:281-289, 1973. 
[72] R. Schori, Inverse limits and homogeneity, Trans. Amer. Math. Soc., 124:533-539, 1966.

[73] W. Sierpiński, Sur une courbe Cantorienne qui contient une image biunivoque et continue de toute courbe donnée, C.R. Acad. Sci. Paris, 162:629-632, 1916.

[74] D. Sullivan, Solenoidal manifolds, J. Singul., 9:203-205, 2014.

[75] D. van Dantzig, Über topologisch homogene Kontinua, Fund. Math., 15:102-125, 1930.

[76] W. van Limbeek, Towers of regular self-covers and linear endomorphisms of tori, Geom. Topol., 22:2427-2464, 2018.

[77] W. van Limbeek, Structure of normally and finitely non-co-Hopfian groups, preprint, arXiv:1710.02179.

[78] A. Verjovsky, Commentaries on the paper Solenoidal manifolds by Dennis Sullivan, J. Singul., 9:245-251, 2014.

[79] L. Vietoris, Über den höheren Zusammenhang kompakter Räume und eine Klasse von zusammenhangstreuen Abbildungen, Math. Ann., 97:454-472, 1927.

[80] S.-C. Wang and Y.Q. Wu, Covering invariants and co-Hopficity of 3-manifold groups, Proc. London Math. Soc. (3), 68:203-224, 1994.

[81] G. Willis, The scale and tidy subgroups for endomorphisms of totally disconnected locally compact groups, Math. Ann., 361:403-442, 2015.

Alex Clark, School of Mathematical Sciences, Queen Mary University of London, London E1 4NS, UK

E-mail address: alex.clark@qmul.ac.uk

Steven Hurder, Department of Mathematics, University of Illinois at Chicago, 322 SeO (m/c 249 ), 851 S. Morgan Street, Chicago, IL 60607-7045

E-mail address: hurder@uic.edu

Olga Lukina, Department of Mathematics, University of Illinois at Chicago, 322 SeO (m/c 249 ), 851 S. Morgan Street, Chicago, IL 60607-7045

E-mail address: ollukina940@gmail.com 